Accepted for publication in The Astrophysical Journal: August 15, 2011

Preprint typeset using LATEX style emulateapj v. 8/13/10

\title{
OPTICAL PROPERTIES OF HOST GALAXIES OF EXTRAGALACTIC NUCLEAR WATER MASERS
}

\author{
Guangtun Zhu ${ }^{1}$, Ingyin Zaw ${ }^{2,1}$, Michael R. Blanton ${ }^{1}$, and Lincoln J. Greenhill ${ }^{3}$ \\ Accepted for publication in The Astrophysical Journal: August 15, 2011
}

\begin{abstract}
We study the optical properties of the host galaxies of nuclear $22 \mathrm{GHz}(\lambda=1.35 \mathrm{~cm})$ water masers. To do so, we cross-match the galaxy sample surveyed for water maser emission (123 detections and 3806 non-detections) with the SDSS low-redshift galaxy sample $(z<0.05)$. Out of 1636 galaxies with SDSS photometry, we identify 48 detections; out of the 1063 galaxies that also have SDSS spectroscopy, we identify 33 detections. We find that maser detection rate is higher at higher optical luminosity $\left(M_{B}\right)$, larger velocity dispersion $(\sigma)$, and higher [O III] $\lambda 5007$ luminosity, with [O III] $\lambda 5007$ being the dominant factor. These detection rates are essentially the result of the correlations of isotropic maser luminosity with all three of these variables. These correlations are natural if maser strength increases with central black hole mass and the level of AGN activity. We also find that the detection rate is higher in galaxies with higher extinction. Based on these results, we propose that maser surveys seeking to efficiently find masers should rank AGN targets by extinction-corrected [O III] $\lambda 5007$ flux when available. This prioritization would improve maser detection efficiency, from an overall $\sim 3 \%$ without pre-selection to $\sim 16 \%$ for the strongest intrinsic [O III] $\lambda 5007$ emitters, by a factor of $\sim 5$.

Subject headings: galaxies: active — galaxies: nuclei — galaxies: Seyfert — radio lines: galaxies — masers
\end{abstract}

\section{INTRODUCTION}

Water maser emission at $22 \mathrm{GHz}(\lambda=1.35 \mathrm{~cm})$ is currently the only tracer of warm dense molecular gas in the inner parsec of active galaxy nuclei (AGNs) and has been detected to date in more than 100 AGNs (e.g., Braatz et al. 1996; Henkel et al. 2005; Kondratko et al. 2006b; Braatz \& Gugliucci 2008; Greenhill et al. 2008). Some of these masers are associated with rotating, highly inclined disk structures close to the central engines ("disk masers") and have been used for a broad variety of astrophysical studies, including the mass estimation of supermassive black holes, the mapping of accretion disks, and the determination of geometric distances (e.g., Mivoshi et al. 1995; Greenhill et al. 1997; Greenhill \& Gwinn 1997; Ishihara et al. 2001; Greenhill et al. 2003; Braatz et al. 2010; Kuo et al. 2010).

Nuclear water masers have been claimed to be associated with Seyfert 2 or low-ionization nuclear emissionline region (LINER) systems (e.g., Braatz et al. 1997; Kondratko et al. 2006b). It is also plausible that AGNs which host masers are more likely associated with high X-ray obscuring columns $\left(N_{H}\right)$ than those without maser detections (e.g., Braatz et al.|1997; Madeiski et al.|2006; Zhang et al. 2006; Greenhill et al. 2008; Zhang et al. 2010). There also appear to be correlations of isotropic maser luminosity with the X-ray luminosity (Kondratko et al. 2006b) and the far-infrared (FIR) luminosity (Henkel et al. 2005) of the host AGNs, though the underlying mechanisms are not clear. As additional words of caution in interpreting these correlations, the in-

\footnotetext{
${ }^{1}$ Center for Cosmology and Particle Physics, Department of Physics, New York University, 4 Washington Place, New York, NY 10003, gz323@nyu.edu

2 New York University Abu Dhabi, P.O. Box 903, New York, NY 10276

${ }^{3}$ Harvard-Smithsonian Center for Astrophysics, 60 Garden Street, Cambridge, MA 02138
}

ferred X-ray luminosities are subject to large uncertainties owing to high columns, and the true (i.e., beamed) maser luminosities are unknown in most cases.

The overall detection rate of nuclear water masers is only $\sim 3 \%$. Even if AGNs with higher X-ray luminosity and/or higher obscuring column more likely host masers, there is no existing large sample of AGNs with X-ray data available for target selection. However, if masers are preferentially found in galaxies with certain optical properties, we can improve maser detection efficiency by selecting galaxies with these properties as targets from existing large galaxy surveys, such as the Sloan Digital Sky Survey (SDSS; York et al. 2000), the 2dF Galaxy Redshift Survey (2dFGRS; Colless et al. 2001), and the 6dF Galaxy Survey (6dFGS; Jones et al. 2004).

The goal of this work is to systematically investigate the optical properties of maser host galaxies. We cross-match the SDSS low-redshift galaxy catalog with the complete galaxy sample surveyed for maser emission. We find that maser detection rate is higher at higher optical luminosity, larger velocity dispersion, higher [O III] $\lambda 5007$ luminosity, and higher extinction. We present these results in Section 2. In Section 3. we suggest that a plausible explanation of these results is that maser strength is correlated with the central black hole mass and the AGN activity of the host galaxies. In Section 4. we suggest that maser surveys rank AGN targets by extinction-corrected [O III] $\lambda 5007$ flux, which should greatly improve the detection efficiency. We adopt a $\Lambda$ CDM cosmology with $\Omega_{\mathrm{m}}=0.3, \Omega_{\Lambda}=0.7$ and $H_{0}=70 \mathrm{~km} \mathrm{~s}^{-1} \mathrm{Mpc}^{-1}$.

\section{DETECTION EFFICIENCY}

\subsection{Data}

2.1.1. The complete galaxy sample surveyed for maser emission 


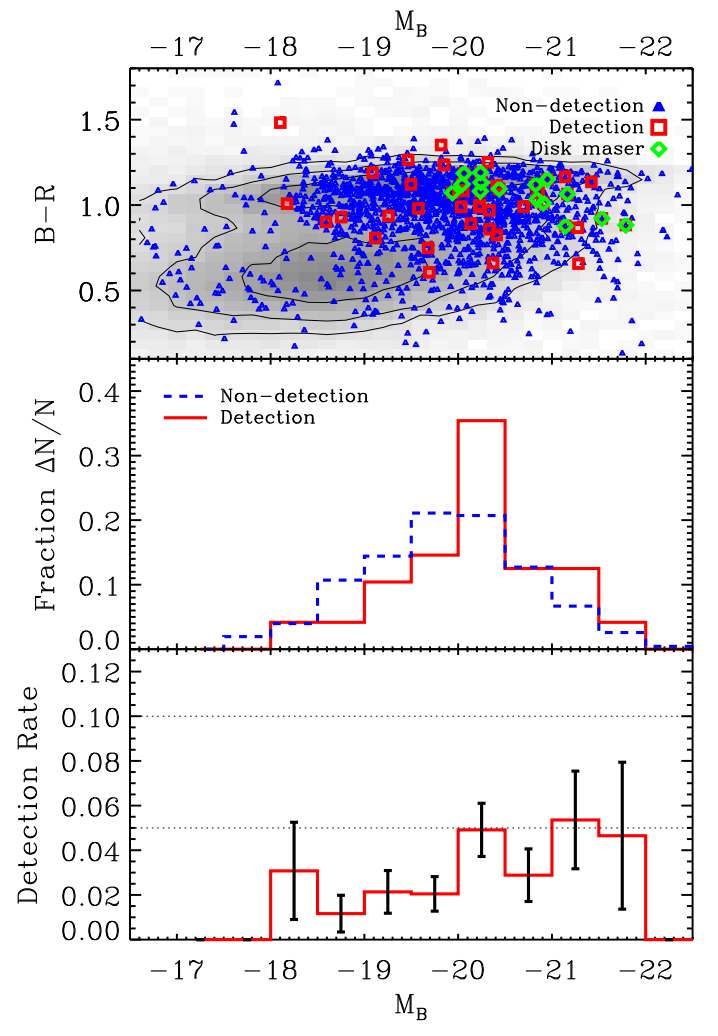

Fig. 1.- Top panel: Color-magnitude diagram. For comparison, we show the distribution of the whole low-z photometric sample as the gray scale. The contours enclose $40 \%, 80 \%$, and $90 \%$ of the sample. Blue triangles represent non-detections and red squares indicate maser detections. We also show the disk masers with green diamonds but we cannot draw robust conclusions because of the small sample size. Middle panel: Distribution of $M_{B}$. We show the ratio of the number of galaxies per $0.5 \mathrm{mag}$ to the size of each sample. Bottom panel: Detection rate as a function of $M_{B}$. To guide the eye, we show two horizontal dotted lines at $5 \%$ and $10 \%$. The error bars represent Poisson errors. The detection rate appears to be higher at higher luminosity.

To construct a complete sample of galaxies surveyed to date for maser emission, we combine the catalogs (as of December 1, 2010) maintained on the website of the Megamaser Cosmology Project (MCP 4 ) and that of the Hubble Constant Maser Experiment (HoME可). For maser detections, we use the MCP catalog that is complete. We however exclude those masers known to be associated with star-forming regions (IC 10, M 33, IC 342, M 82, NGC 253, NGC 3359, NGC 3556, NGC 2146, He 2-10, NGC 4038/39, NGC 4214, NGC 5253), as noted in the either HoME or MCP catalogs. Although it is not labeled in either catalog, we also exclude NGC 4194, since it is an ongoing merger and has strong star formation in the center (e.g., Balzano 1983) and the detected maser may not be associated with its nucleus. There are 123 detections in total and we list them in Table 1. Among these, at least 41 are probably associated with disk structures, as noted in the HoME catalog. The evidence is either from direct mapping of the emission distributions using Very Long Baseline Interferometry (VLBI)

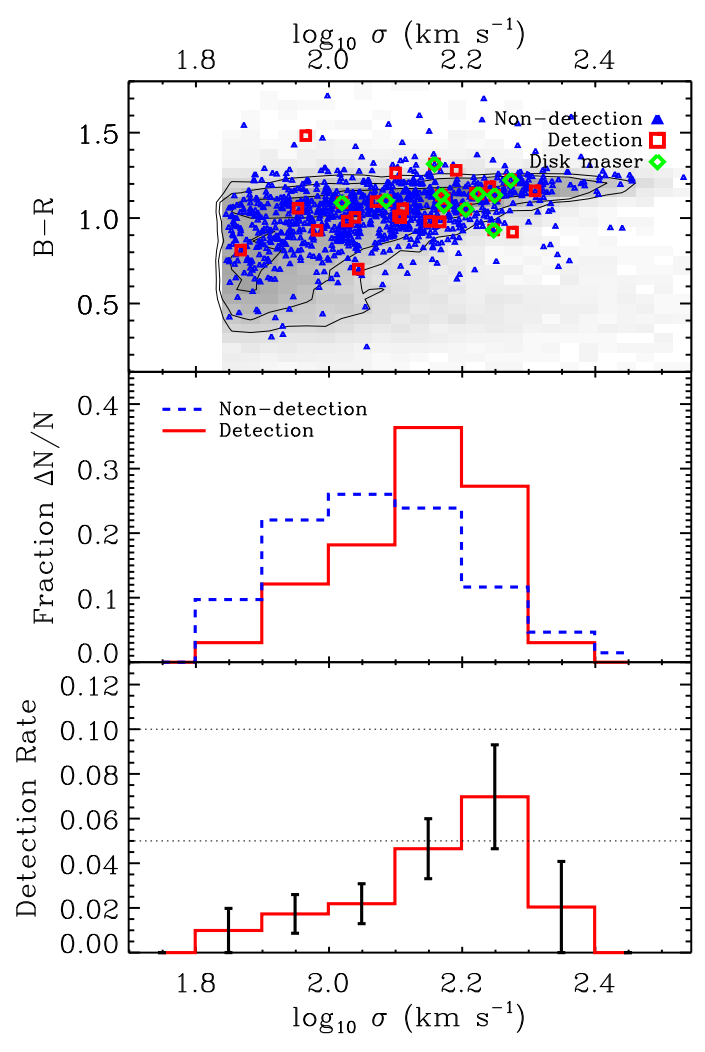

FIG. 2.- Similar to Figure 1 but with velocity dispersion $(\sigma)$. Note $\sigma$ lower than $70 \mathrm{~km} \mathrm{~s}^{-1}$ is not reliable due to the instrumental resolution of the SDSS spectrograph; we do not consider galaxies below that limit. The detection rate is higher at larger $\sigma$.

or inferred from spectroscopy (e.g., Madejski et al. 2006; Greenhill et al. 2008). For galaxies without successful maser detections (non-detections), we combine both catalogs from HoME and MCP to build the whole sample. After removing duplicates between the catalogs, we have 3806 non-detections in total. The overall detection rate is therefore about $3 \%$.

We note that this complete sample consists of galaxies surveyed using different telescopes with different detection sensitivities. The highest sensitivity comes from the Green Bank Telescope (GBT) survey (e.g., Braatz et al. 2004). The $1 \sigma \mathrm{rms}$ sensitivity of the GBT survey is $\sim 3$ mJy per $24.4 \mathrm{kHz}\left(\sim 0.33 \mathrm{~km} \mathrm{~s}^{-1}\right)$ channel (Braatz et al. 2004). Assuming a characteristic maser linewidth of $10 \mathrm{~km} \mathrm{~s}^{-1}$, this corresponds to a $3 \sigma$ maser flux limit of $0.1 \mathrm{Jy} \mathrm{km} \mathrm{s}^{-1}$.

\subsubsection{The SDSS low-z galaxy sample}

To systematically study maser detection efficiency, we require a complete parent sample. The SDSS survey has provided such a sample of galaxies with uniform imaging and spectroscopy. For spectral properties, we use the measurements by the MPA-JHU groun6 (e.g., Tremonti et al. 2004). We use the latest version that corresponds to SDSS Data Release 7 (DR7, Abazajian et al. 2009). We choose to look at velocity dispersion $(\sigma$, in $\left.\mathrm{km} \mathrm{s}^{-1}\right)$ and [O III] $\lambda 5007$ luminosity $\left(L_{[\mathrm{OIII}] \lambda 5007}\right.$, in

\footnotetext{
4 https://safe.nrao.edu/wiki/bin/view/Main/MegamaserCosmologyProject

${ }^{5}$ https://www. cfa.harvard.edu/ lincoln/demo/HoME/index.html 6 http://www.mpa-garching.mpg.de/SDSS/DR7/
} 


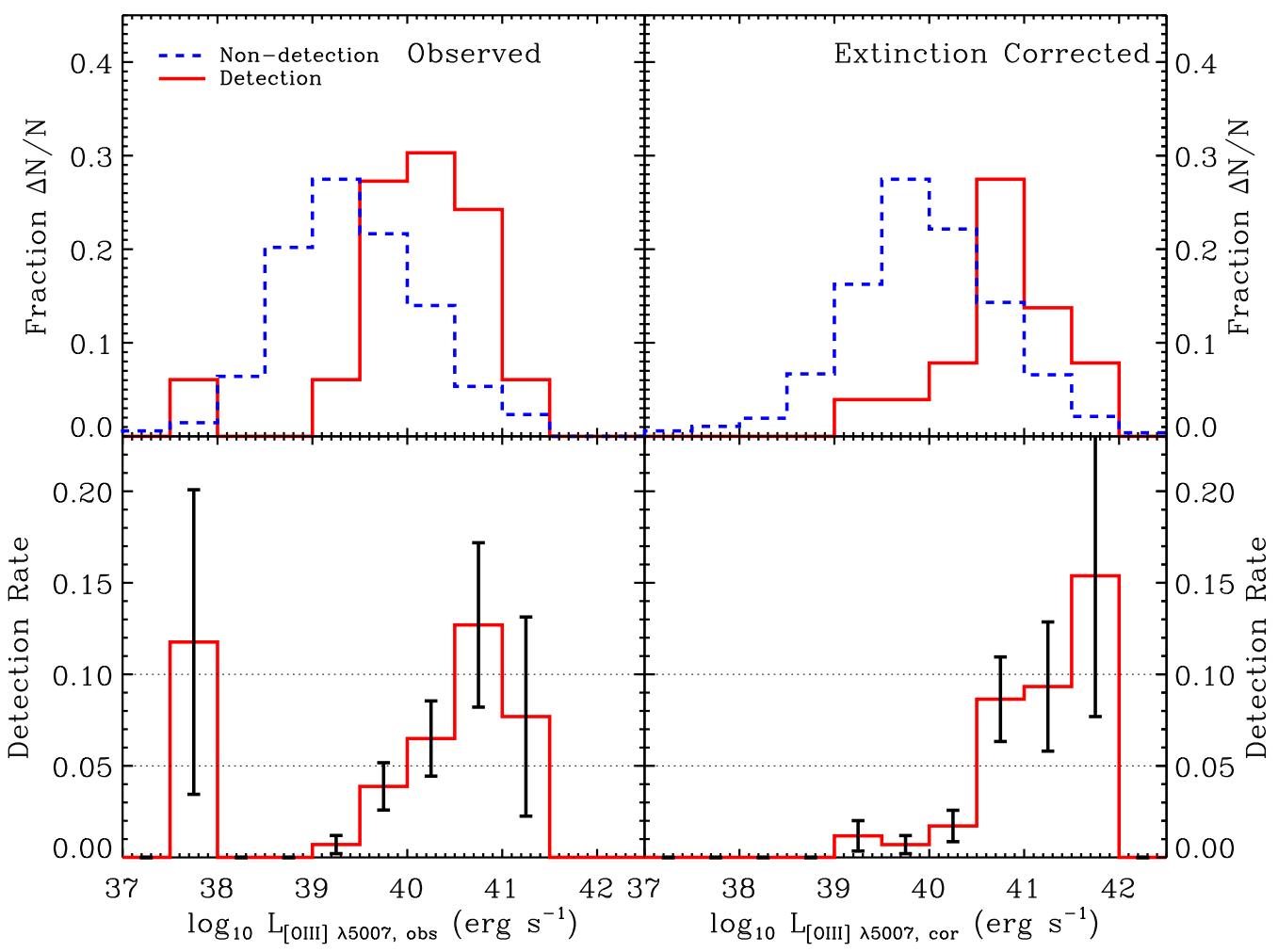

FIG. 3. - The left panels are similar to the lower two panels in Figure 1 and 2 but with observed [O III] $\lambda 5007$ luminosity $\left(L_{[\mathrm{OIII}] \lambda 5007, \mathrm{obs}}\right)$. In the right panels, we correct the extinction to obtain the intrinsic [O III $] \lambda 5007$ luminosity $\left(L_{[\mathrm{OIII}] \lambda 5007, \mathrm{cor}}\right)$ using $L_{[\mathrm{OIII}] \lambda 5007, \mathrm{cor}}=$ $L_{[\mathrm{OIII}] \lambda 5007 \text {,obs }} /\left((\mathrm{H} \alpha / \mathrm{H} \beta) /(\mathrm{H} \alpha / \mathrm{H} \beta)_{0}\right)^{2.94}$, where $\mathrm{H} \alpha / \mathrm{H} \beta$ is the observed Balmer decrement and we assume the intrinsic Balmer decrement $(\mathrm{H} \alpha / \mathrm{H} \beta)_{0}=3$. The detection rate is higher at higher [O III] $\lambda 5007$ luminosity. The effect is stronger for $L_{[\mathrm{OIII}] \lambda 5007 \text {,cor }}$, implying that detections on average have higher extinction than non-detections.

erg $\mathrm{s}^{-1}$ ), since velocity dispersion is closely related to central black hole mass (e.g., Ferrarese \& Merritt 2000; Gebhardt et al. 2000) and [O III] $\lambda 5007$ luminosity is well-correlated with AGN activity (e.g., Heckman et al. 2005). It is well-known that [O III] $\lambda 5007$ can be severely obscured by material in the host galaxy (e.g., Diamond-Stanic et al. 2009). We therefore calculate the intrinsic $\left[\begin{array}{ll}\mathrm{O} & \mathrm{III}\end{array}\right] \lambda 5007$ luminosity $\left(L_{[\mathrm{OIII}] \lambda 5007, \mathrm{cor}}\right)$ by correcting the observed $L_{[\mathrm{OIII}] \lambda 5007 \text {,obs }}$ using the following formula (e.g., Bassani et al. 1999): $L_{[\mathrm{OIII}] \lambda 5007, \mathrm{cor}}=$ $L_{[\mathrm{OIII}] \lambda 5007, \mathrm{obs}} /\left((\mathrm{H} \alpha / \mathrm{H} \beta) /(\mathrm{H} \alpha / \mathrm{H} \beta)_{0}\right)^{2.94}$, where $\mathrm{H} \alpha / \mathrm{H} \beta$ is the observed Balmer decrement and we assume the intrinsic Balmer decrement $(\mathrm{H} \alpha / \mathrm{H} \beta)_{0}=3$. Because of the instrumental dispersion of the SDSS spectrograph, velocity dispersion measurements smaller than $70 \mathrm{~km} \mathrm{~s}^{-1}$ are not reliable7; we thus only consider galaxies with $\sigma>70 \mathrm{~km} \mathrm{~s}^{-1}$. Finally, since only four $(4 \mathrm{C}+05.19$, SDSS J0804+3607, Mrk 34, and 3C 403) out of the 123 maser detections are farther than $z=0.05$ and all of them are not in the MPA-JHU catalog, we limit the sample to low-redshift galaxies with $z<0.05$. At the faint end, the flux limit of the SDSS spectroscopic survey is $r=17.77$, which corresponds to $M_{B} \sim-18$ at $z=0.05$.

Due to the difficulty of automatic photometric processing of big galaxies, the SDSS catalog is missing many nearby, bright galaxies, even though they are contained within the SDSS imaging footprint. For photometry, we therefore use the low-z catalog $(z<0.05)$ from the NYU Value Added Galaxy Catalog (NYU-VAGC8; Blanton et al. 2005). This low-z photometric catalog includes any low-redshift galaxies from the Third Reference Catalog of Bright Galaxies (RC3; de Vaucouleurs et al. 1991; Corwin et al. 1994) for which we have ugriz imaging from SDSS, but which are not in the SDSS catalog. We use the latest version of this catalog that corresponds to SDSS Data Release 6 (DR6, Adelman-McCarthy et al. 2006). We have compared the photometry of those galaxies in the SDSS catalog with that from DR7 and found they are very consistent, therefore using DR6 for photometry should not introduce any bias. We derive absolute magnitudes using the kcorrect package (v4.1.4; Blanton \& Roweis 2007). For easier comparison with previous studies, we choose the $B$ band magnitude $M_{B}$ to indicate optical luminosity. Note here the magnitude is the total magnitude for the whole galaxy.

We cross-match maser detections and non-detections with the SDSS low-z sample, and identify 48 detections (15 disk masers) and 1588 non-detections with SDSS photometry, among which, 33 detections (10 disk masers) and 1030 non-detections have reliable spectral measure-

8 http://sdss.physics.nyu.edu/vagc/

\footnotetext{
7 http://www.sdss.org/dr7/algorithms/veldisp.html
} 


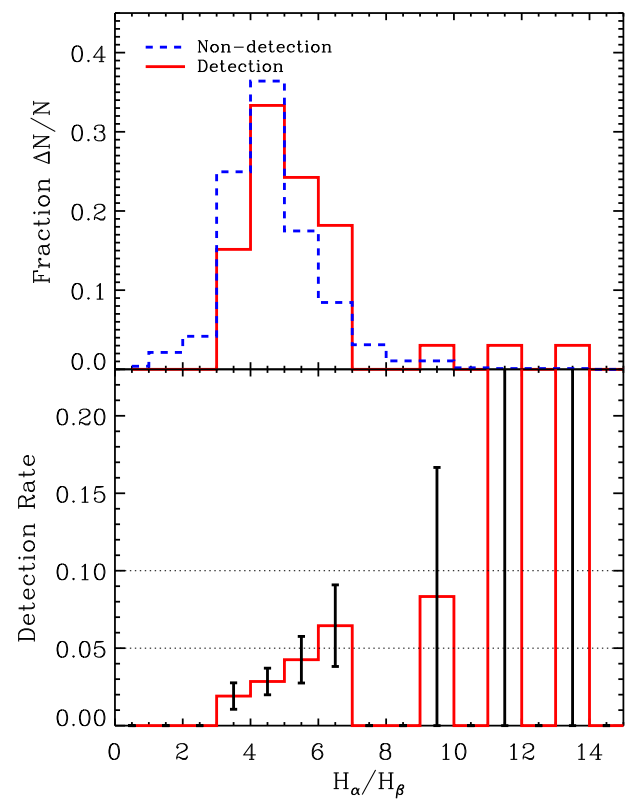

FIG. 4. - Similar to the lower two panels in Figure 1 and 2 but with Balmer decrement $\mathrm{H} \alpha / \mathrm{H} \beta$. The detection rate is higher at higher $\mathrm{H} \alpha / \mathrm{H} \beta$.

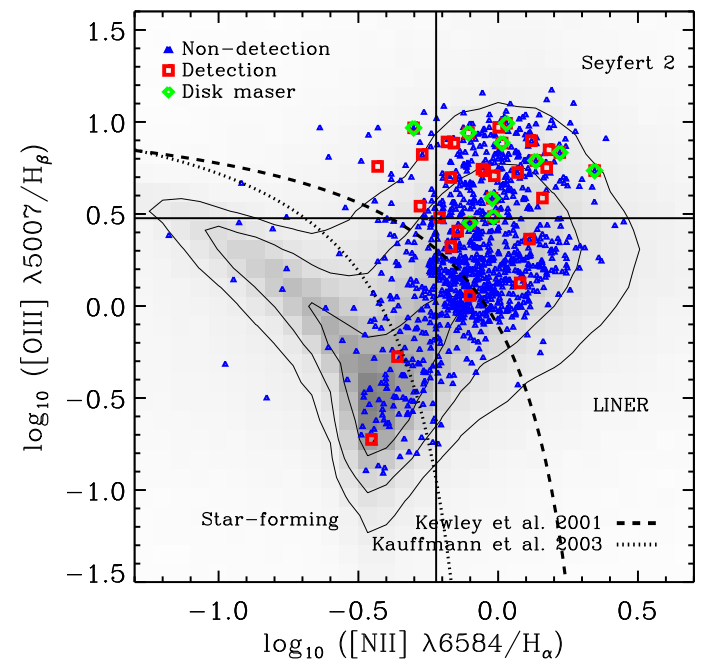

FIG. 5.- Emission line diagnostic diagram (Baldwin et al. 1981, BPT). For comparison, we show the distribution of the whole low$\mathrm{z}$ spectroscopic sample as the gray scale. The contours enclose $40 \%, 80 \%$, and $90 \%$ of the sample. The dotted and dashed lines are the demarcation lines separating AGN and star-forming galaxies defined by Kauffmann et al. (2003) and Kewley et al. (2001). The solid vertical and horizontal lines at [N II] $\lambda 6584 / \mathrm{H} \alpha=0.6$ and [O III $] \lambda 5007 / \mathrm{H} \beta=3.0$ are the conventional separating lines for Seyfert 2 galaxies (above) and LINERs (below). There are 25 (8) detections above (below) the horizontal line, compared to 296 (734) non-detections. ments, i.e., with $\sigma>70 \mathrm{~km} \mathrm{~s}^{-1}$. We note that the overall detection rate in this sample is $\sim 3 \%$, the same as in the total sample, so using this sample should not introduce a bias in the analysis presented below. We present measurements for detections in Table 1. In the next subsection, we study the dependence of maser detection efficiency on optical luminosity and spectral properties using these samples.

\subsection{Results}

\subsubsection{All galaxies surveyed for water masers}

Figure 1, 2, and 3] present maser detection efficiency as a function of $M_{B}, \sigma$ (in log scale), $L_{[\mathrm{OIII}] \lambda 5007 \text {,obs }}$ (in

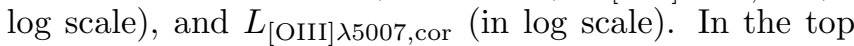
panels of Figure 1 and 2, we show the detections (blue triangle) and non-detections (red square) in the colormagnitude $/ \sigma$ diagram. For comparison, we also show the distribution of the whole SDSS low-z sample in gray scales and contours. Masers are concentrated in systems with larger $B-R$ and total luminosity, but there are very few blue, low luminosity (presumably disk-dominated) galaxies that have been surveyed for emission. We also show the disk masers with green diamonds, but we cannot draw robust conclusions because of the small sample size. In the middle panels of Figure 1 and 2 and in the top panels of Figure 3. we show the distribution of detections and non-detections. Performing KolmogorovSmirnov test yields P-values $0.01,0.02,2 \times 10^{-8}$, and $3 \times 10^{-9}$, respectively, indicating a low probability that they are drawn from the same distribution, particularly for $L_{[\mathrm{OIII}] \lambda 5007}$. The bottom panels present the detection rate, which is apparently higher at brighter $M_{B}$, larger $\sigma$, and higher $L_{[\mathrm{OIII}] \lambda 5007}$. The dependence of the detection efficiency on $\sigma$ appears stronger than that on $M_{B}$, while the dependence on $L_{[\mathrm{OIII}] \lambda 5007}$ is more striking than that on both $M_{B}$ and $\sigma$.

Figure 3 also shows that maser detection efficiency depends more strongly on $L_{[\mathrm{OIII}] \lambda 5007 \text {,cor than on }}$ $L_{[\mathrm{OIII}] \lambda 5007, \mathrm{obs}}$, implying that detections could on average have higher extinction than non-detections. Previous studies show that AGNs which host masers are more likely associated with high X-ray obscuring columns than those without maser detections (e.g., Greenhill et al. 2008; Zhang et al. 2010). We therefore investigate the detection efficiency as a function of the observed Balmer decrement in Figure 4. In the top panel, a KolmogorovSmirnov test yields a $\mathrm{P}$-value 0.009 , indicating that detections and non-detections are likely drawn from different distributions. The lower panel shows the detection rate is indeed higher at higher extinction.

In Figure 5. we show the emission line diagnostic diagram (Baldwin et al. 1981, BPT). We show two widely-used demarcation criteria that separate AGNs (to the right) and star-forming galaxies (to the left), by Kewley et al. (2001, the dashed line) and by Kauffmann et al. (2003, the dotted line). The vertical solid line at $[\mathrm{N}$ II $] \lambda 6584 / \mathrm{H} \alpha=0.6$ and the horizontal solid line at [O III] $\lambda 5007 / \mathrm{H} \beta=3.0$ are the conventional demarcation lines for Seyfert 2 (above) and LINER-like (below) galaxies (e.g., Veilleux \& Osterbrock 1987). It is apparent that most surveys have mainly targeted AGNs.

Among AGNs, masers are clearly more often detected in Seyfert 2 galaxies rather than LINERs. For exam- 


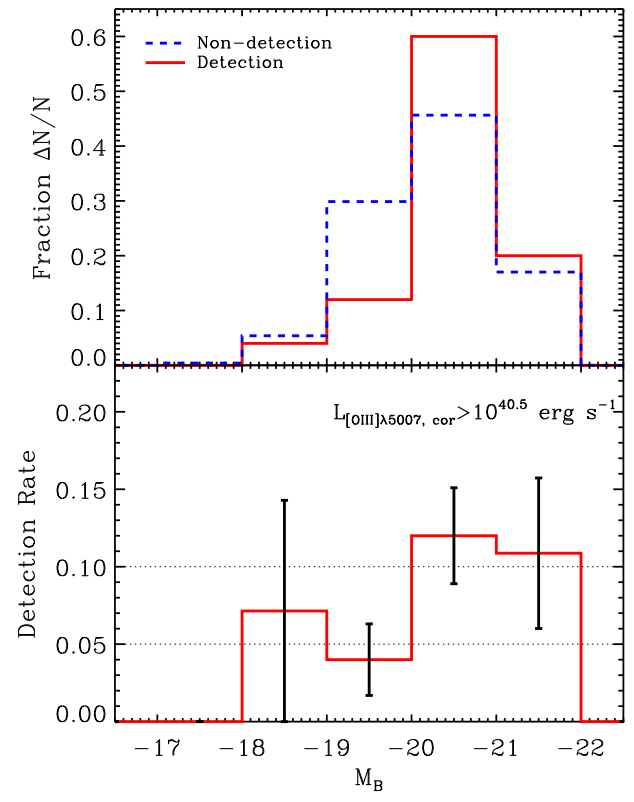

FIG. 6. - The same as the lower two panels in Figure 1 but

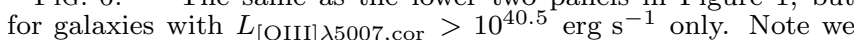
double the binsize because of a smaller sample size. Among the 33 detections, 25 have $L_{[\mathrm{OIII}] \lambda 5007 \text {,cor }}>10^{40.5} \mathrm{erg} \mathrm{s}^{-1}$; and among 1030 non-detections, 242 have $L_{[\mathrm{OIII}] \lambda 5007, \mathrm{cor}}>10^{40.5} \mathrm{erg} \mathrm{s}^{-1}$. The overall detection rate $(\sim 9 \%)$ is higher than that $(\sim 3 \%)$ without pre-selection in Figure 1 The detection rate is also higher at higher luminosity.

ple, in Figure [5] there are 25 maser detections associated with Seyfert 2 galaxies (out of 321 in total, above the horizontal line), but only eight associated with LINERs (out of 742 in total, below the horizontal line). It is yet unkown what fraction of LINERs are AGNs (e.g., Ho et al. 2003; Sarzi et al. 2010) and we are not sure about the underlying mechanisms responsible for the preferred association of masers with Seyfert 2 galaxies over LINERs. However, if LINERs are low-luminosity counterparts of Seyfert 2 galaxies, as some studies claim (e.g., Ho et al. 2003), then the low maser detection efficiency among LINERs could be simply a reflection of the $L_{[\mathrm{OIII}] \lambda 5007}$ dependence we found in Figure 3, since the majority of LINERs without maser detections ( 90\%) have $L_{[\mathrm{OIII}] \lambda 5007 \text {,cor }}<10^{40.5} \mathrm{erg} \mathrm{s}^{-1}$. After all, LINERs are known to have low [O III] $\lambda 5007$ luminosities relative to Seyfert 2 galaxies (e.g., Heckman et al. 2004).

\subsubsection{Galaxies with $L_{[\mathrm{OIII}] \lambda 5007, \mathrm{cor}}>10^{40.5} \mathrm{erg} \mathrm{s}^{-1}$ only}

We have shown that maser detection rate depends more strongly on $L_{[\mathrm{OIII}] \lambda 5007}$ than on $M_{B}$ and $\sigma$, as in Figures 11. 2] and 3. However, these three quantities are themselves correlated in the galaxy population. We here investigate further whether there is a residual dependence of detection efficiency on $M_{B}$ and $\sigma$ even for galaxies with strong [O III] $\lambda 5007$ emission.

Among the 33 detections, only eight have $L_{\text {[OIII] } \lambda 5007, \text { cor }}<10^{40.5} \mathrm{erg} \mathrm{s}^{-1}$. Meanwhile, 789 out of 1030 non-detections have $L_{[\mathrm{OIII}] \lambda 5007 \text {,cor }<}$ $10^{40.5} \mathrm{erg} \mathrm{s}^{-1}$. We therefore pre-select galaxies with $L_{[\mathrm{OIII}] \lambda 5007, \mathrm{cor}}>10^{40.5} \mathrm{erg} \mathrm{s}^{-1}(25 / 33$ detections and

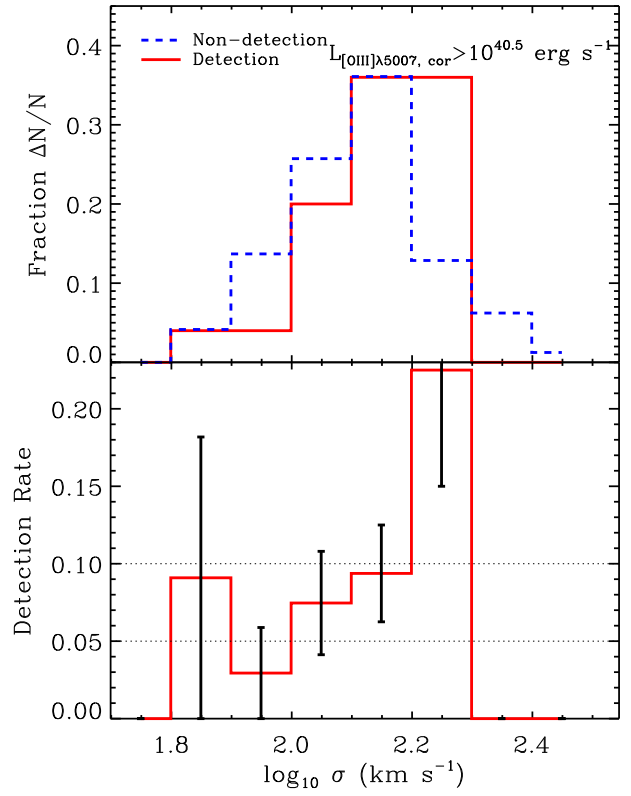

Fig. 7. - The same as the lower two panels in Figure 2 but for galaxies with $L_{[\mathrm{OII}] \lambda 5007 \text {,cor }}>10^{40.5} \mathrm{erg} \mathrm{s}^{-1}$ only. The overall detection rate is higher than without pre-selection in Figure 2 The detection rate is higher at larger $\sigma$.

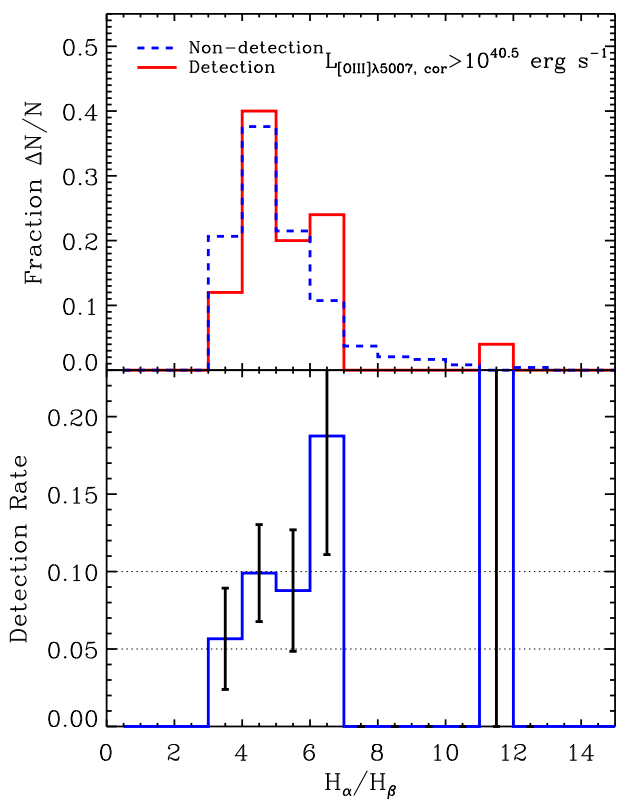

Fig. 8.- The same as the lower two panels in Figure 4 but for galaxies with $L_{[\mathrm{OIII}] \lambda 5007 \text {,cor }}>10^{40.5} \mathrm{erg} \mathrm{s}^{-1}$ only. The overall detection rate is higher than without pre-selection in Figure 4 The detection rate is higher at higher $\mathrm{H} \alpha / \mathrm{H} \beta$. 
$242 / 1030$ non-detections) and show the detection rate as a function of $M_{B}$ and $\sigma$ for this pre-selected sample in Figure 6 and 7 . We also show the detection rate as a function of Balmer decrement $\mathrm{H} \alpha / \mathrm{H} \beta$ in Figure 8 . Compared to the results for the whole sample (Figure 1. 2. and 4), there are still detections over the whole range of $M_{B}, \sigma$, and $\mathrm{H} \alpha / \mathrm{H} \beta$, and the detection rate is still higher at brighter $M_{B}$, larger $\sigma$, and higher $\mathrm{H} \alpha / \mathrm{H} \beta$. The overall detection rate, however, is $\sim 9 \%$ compared to $\sim 3 \%$ without pre-selection. We therefore conclude that among $M_{B}, \sigma$, [O III] $\lambda 5007$, and $\mathrm{H} \alpha / \mathrm{H} \beta$, [O III] $\lambda 5007$ is the dominant factor when determining maser detection efficiency.

\section{DISCUSSION}

\subsection{Isotropic luminosity of masers}

Above, we found that water maser detection rate increases with [O III $\lambda$ $\lambda 5007$ luminosity $\left(L_{[\mathrm{OIII}] \lambda 5007}\right)$, velocity dispersion $(\sigma)$, and optical luminosity $\left(M_{B}\right)$. We speculate that these correlations may be a consequence of an underlying correlation of these parameters with water maser luminosity. The true luminosity of masers is difficult to measure because the maser emission is likely to be beamed (e.g., Elitzur 1992) and estimates of the beaming angle require a detailed model of the maser, which can only be inferred in the cases with wellunderstood geometries from VLBI observations (e.g., Miyoshi et al. 1995). In place of true luminosity, we adopt apparent luminosity, which is based on the premise of "isotropic" emission of radiation. The isotropic luminosity can be computed readily from the flux densities observed in spectra. On the other hand, the flux density can be variable on time scales of months (e.g., Bragg et al. 2000; Braatz et al. 2003; Herrnstein et al. 2005; Castangia et al. 2008), which introduces another uncertainty. Nonetheless, analysis using the stand-in of isotropic luminosity provides an opportunity to investigate whether the speculated correlations exist. From the literature, we have collected isotropic luminosities for 66 masers.

Maser surveys are flux-limited and the lower maser detection rate in galaxies with lower $L_{[\mathrm{OIII}] \lambda 5007, \mathrm{obs}}$, smaller $\sigma$, and fainter $M_{B}$ could be because most masers in these galaxies are too faint to be detected. We investigate the flux limit in Figure 9, where we plot isotropic luminosities as a function of redshift. Although these detections are from a variety of surveys that have different sensitivities, they appear to be consistent with $0.1 \mathrm{Jy} \mathrm{km} \mathrm{s}^{-1}$ as the effective limit. This limit is consistent with a plausible detection threshold of $10 \mathrm{mJy}(3 \sigma)$ in a $1 \mathrm{~km} \mathrm{~s}^{-1}$ channel and blends of Doppler components on the order of $10 \mathrm{~km} \mathrm{~s}^{-1}$, as seen in the spectra (e.g., with GBT, Braatz et al. 2004).

To investigate the correlations between maser luminosity and optical properties of the host galaxies, we comple-

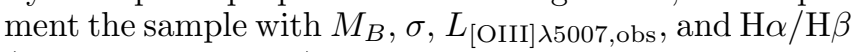
(thus $L_{[\mathrm{OIII}} \lambda 5007$,cor $)$ from the literature. In addition to those with SDSS photometry or spectroscopy, we have compiled $L_{[\mathrm{OIII}] \lambda 5007 \text {, cor }}(\mathrm{H} \alpha / \mathrm{H} \beta), L_{[\mathrm{OIII}] \lambda 5007, \text { obs }}, \sigma$, and $M_{B}$ for $36,40,43$, and 54 masers with measured isotropic luminosities. We present these data in Table 1. The combined sample is inhomogeneous in terms of selection, technique, and instrument parameters, and this (in ad-

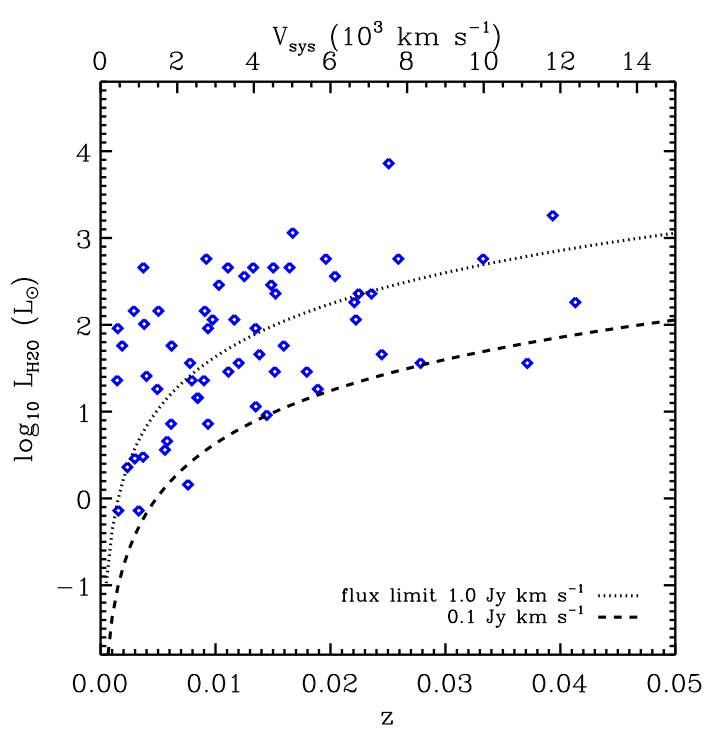

FIG. 9.- Isotropic maser luminosity as a function of redshift for 66 extragalactic nuclear masers. The dotted line shows the flux limit $1.0 \mathrm{Jy} \mathrm{km} \mathrm{s}^{-1}$, and the dashed line shows the flux limit 0.1 Jy $\mathrm{km} \mathrm{s}^{-1}$.

dition to the use of isotropic luminosity as a stand-in noted earlier) may be expected to increase the scatter in any correlations.

In Figure 10 we plot $\log _{10} L_{[\mathrm{OIII}] \lambda 5007, \text { cor }}$, $\log _{10} L_{[\mathrm{OIII}] \lambda 5007, \mathrm{obs}}, \quad \log _{10} \sigma, \quad$ and $M_{B}$ against the isotropic luminosity $\log _{10} L_{\mathrm{H} 2 \mathrm{O}}$. Although the scatter in all relations is relatively large, the variables are clearly correlated. Assuming a uniform error of $0.5 \mathrm{dex}$ for $\log _{10} L_{\mathrm{H} 2 \mathrm{O}}$, we perform least-squares fits with the following linear relations: $\log _{10} L_{\mathrm{H} 2 \mathrm{O}}=a+b\left(x-x_{0}\right)$, where we choose $x_{0}$ to be the medians, 41.39 (dex), 40.43 (dex), 2.16 (dex), and -20.58 (mag)

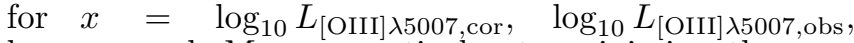
$\log _{10} \sigma$, and $M_{B}$, respectively, to minimize the correlation between $a$ and $b$. We show the results with the dashed lines. The intercepts and slopes $(a, b)$ for $\log _{10} L_{[\mathrm{OIII}] \lambda 5007, \mathrm{cor}}, \log _{10} L_{[\mathrm{OIII}] \lambda 5007, \mathrm{obs}}, \log _{10} \sigma$, and $M_{B}$ are $(1.65 \pm 0.13,0.31 \pm 0.10),(1.66 \pm 0.12,0.22 \pm 0.11)$, $(1.64 \pm 0.11,2.67 \pm 0.64)$, and $(1.75 \pm 0.11,-0.21 \pm 0.13)$, respectively. We can draw a robust conclusion that masers are stronger in hosts with higher [O III] $\lambda 5007 \mathrm{lu}$ minosity, larger $\sigma$, and higher optical luminosity, as implied from the detection efficiency results.

We would like to emphasize that the best-fit slopes and intercepts here should be taken with caution. First, as mentioned above, the isotropic luminosity is a poor indicator of maser strength and the sample of optical properties is not homogeneous. Second, stronger masers are more easily detected, and at the faint end there should be a detection bias favoring stronger masers scattered above the real correlations; the real slopes therefore should be steeper than the best-fit ones. Finally, the sample size is small and a few outliers could significantly affect the fitting. However, the conclusion is robust that maser strength increases with [O III] $\lambda 5007$ luminosity, $\sigma$, and optical luminosity.

In Figure 4, we showed that maser detection rate is 


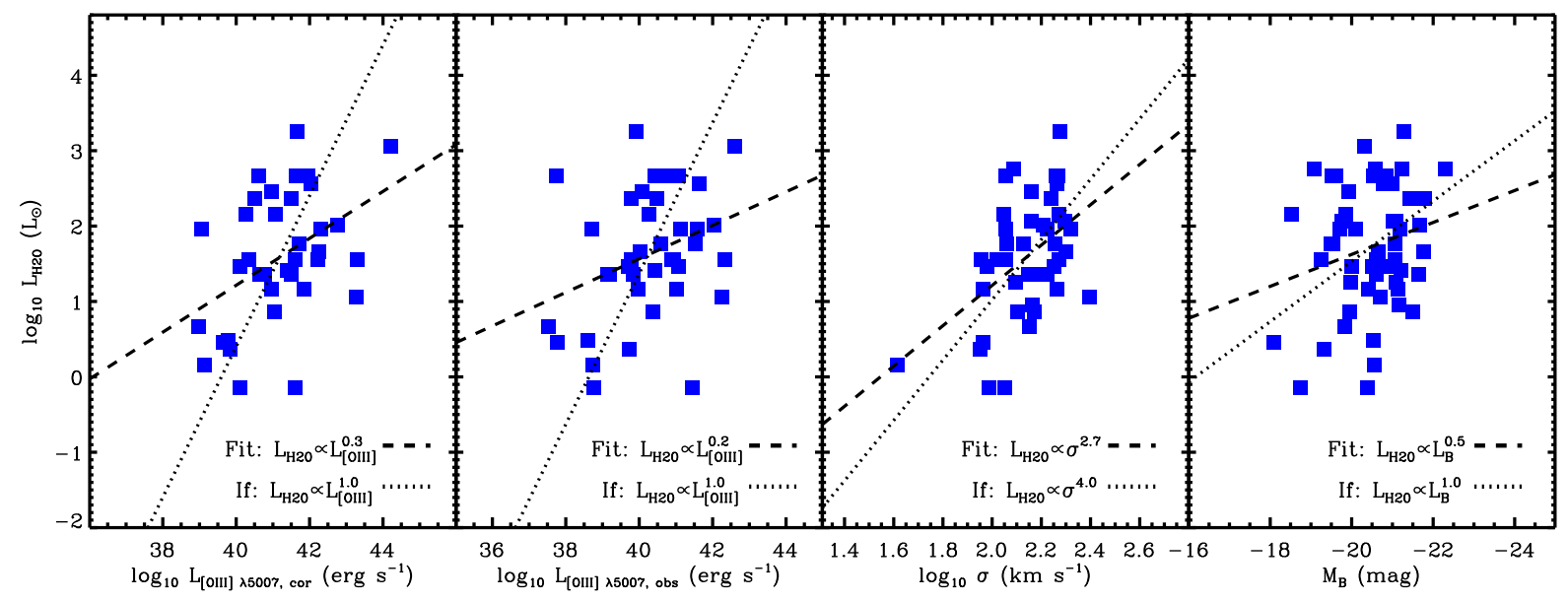

FIG. 10.- Relations between isotropic maser luminosity $\left(L_{\mathrm{H} 2 \mathrm{O}}\right)$ and $L_{[\mathrm{OIII}] \lambda 5007, \mathrm{cor}}, L_{[\mathrm{OIII}] \lambda 5007 \text {,obs }}, \sigma$, and $M_{B}$ of the host galaxies. The dashed lines are the linear least-squares fits assuming a uniform error of 0.5 dex in $\log _{10} L_{\mathrm{H} 2 \mathrm{O}}$. The dotted lines show the linear least-squares fits with the slopes fixed assuming that $L_{\mathrm{H} 2 \mathrm{O}} \propto L_{\mathrm{AGN}} \propto M_{\mathrm{BH}}$, and $M_{\mathrm{BH}} \propto L_{B} \propto \sigma^{4}$, and $L_{\mathrm{AGN}} \propto L_{[\mathrm{OIII}] \lambda 5007}$.

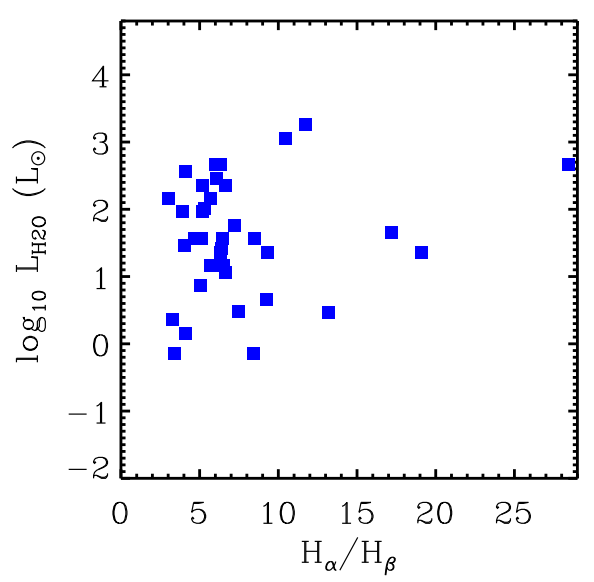

FIG. 11.- Relation between isotropic maser luminosity $\left(L_{\mathrm{H} 2 \mathrm{O}}\right)$ and $\mathrm{H} \alpha / \mathrm{H} \beta$ of the host galaxies.

higher at higher $\mathrm{H} \alpha / \mathrm{H} \beta$. As for the other parameters, this could result from an underlying relation between maser luminosity and extinction. We investigate such a relation in Figure 11. A linear least-squares fit gives $(a, b)$ $=(1.54 \pm 0.15,0.03 \pm 0.03)$ for $x_{0}=6.31$. We do not find a convincing relation (with only $\sim 1 \sigma$ ) between maser luminosity and Balmer decrement, indicating that there might not be a direct linear relation between $\log _{10} L_{\mathrm{H} 2 \mathrm{O}}$ and Balmer decrement. We discuss this result more in Section 3.3

\subsection{Correlations of maser emission with central black hole mass and AGN activity?}

It has long been proposed that water maser emission is closely related with the central black hole. Assuming that a thin viscous accretion disk is obliquely illuminated by a central X-ray source, Neufeld \& Maloney (1995) find that the critical outer radius $R_{\text {cr }}$ at which the disk becomes atomic and the maser emission ceases follows $R_{\text {cr }} \propto L_{2-10}^{-0.43} \dot{m}^{0.81} M_{\mathrm{BH}}^{0.62}$, where $L_{2-10}$ is the $2-10 \mathrm{keV}$ X-ray luminosity of the host galaxy, $\dot{m}$ is the mass accretion rate, and $M_{\mathrm{BH}}$ is the central black hole mass. If we assume that the X-ray luminosity is proportional to AGN bolometric luminosity $\left(L_{\mathrm{AGN}}\right)$, and that $L_{\mathrm{AGN}}$ is proportional to the accretion rate (e.g., Frank, King, \& Raine 2002), we consequently reach $R_{\mathrm{cr}} \propto L_{\mathrm{AGN}}^{0.38} M_{\mathrm{BH}}^{0.62}$. Considering that in the geometrical maser models (e.g. Miyoshi et al. 1995), maser spots do not cover the whole nuclear disk but rather lie on several radial arms, we assume that total maser luminosity $L_{\mathrm{H} 2 \mathrm{O}} \propto R_{\mathrm{cr}}$ (but see Kondratko et al. 2006b who assume $\left.L_{\mathrm{H} 2 \mathrm{O}} \propto R_{\mathrm{cr}}^{2}\right)$. We then expect $L_{\mathrm{H} 2 \mathrm{O}} \propto L_{\mathrm{AGN}}^{0.38} M_{\mathrm{BH}}^{0.62}$. If we further assume that AGNs radiate with a roughly fixed Eddington ratio $\eta$ as a function of black hole mass, so that $L_{\mathrm{AGN}}=\eta L_{\mathrm{Edd}} \propto M_{\mathrm{BH}}$, we eventually reach $L_{\mathrm{H} 2 \mathrm{O}} \propto L_{\mathrm{AGN}} \propto M_{\mathrm{BH}}$.

It is well-known that there exists a tight correlation between the central black hole mass $\left(M_{\mathrm{BH}}\right)$ and the velocity dispersion of the black hole host (Ferrarese \& Merritt 2000; Gebhardt et al. 2000; Tremaine et al. 2002; Gültekin et al. 2009): $M_{\mathrm{BH}} \propto \sigma^{4}$. The optical luminosity also correlates with the black hole mass but with a larger scatter (Magorrian et al. 1998; Gültekin et al. 2009): $M_{\mathrm{BH}} \propto L$ (Optical). For AGN strength, the [O III $\lambda$ is007 luminosity is a reasonably reliable indicator (e.g., Heckman et al. 2005). In Figure 10, assuming $L_{\mathrm{H} 2 \mathrm{O}} \propto M_{\mathrm{BH}} \propto L_{B} \propto \sigma^{4}$ and $L_{\mathrm{H} 2 \mathrm{O}} \propto L_{\mathrm{AGN}} \propto L_{[\mathrm{OIII}] \lambda 5007}$, we fit the intercepts (at 0 ) and obtain $-39.6 \pm 0.2,-38.6 \pm 0.2,-7.0 \pm 0.1$, and $-6.5 \pm 0.1$, for $\log _{10} L_{[\mathrm{OIII}] \lambda 5007, \mathrm{cor}}, \log _{10} L_{[\mathrm{OIII}] \lambda 5007, \mathrm{obs}}$, $\log _{10} \sigma$, and $M_{B}$, respectively. We show these fits with dotted lines. If one takes our best-fit correlations in Section 3.1 at face value, it implies a weaker dependence of maser luminosity on black hole mass and AGN activity than the simplified theory.

The analysis above is simplified. For example, the Eddington ratio $\eta$ is not necessarily independent of black hole mass for masers, even if it is so for AGNs. If masers are powered by AGN luminosity, then a high enough Ed- 
dington ratio is required to pump up a strong maser. This hypothesis can explain what we found in Section 2.2.2, that [O III] $\lambda 5007$ luminosity is more important a factor than $M_{B}$ and $\sigma$ when determining detection efficiency. It is also interesting that the slopes above are all steeper than the best-fit ones. As we discussed in Section 3.1 the best-fit slopes could be flatter than real ones due to the detection bias. Considering the large uncertainties caused by the variability and the assumption of isotropy in maser luminosity and the small sample size, we conclude that our fitting results are in reasonable agreement with the simplified theory and that maser strength is indeed correlated with the central black hole mass and the AGN activity of the host galaxies.

Previous studies have also found correlations between the isotropic luminosity and various properties of the host galaxies. Kondratko et al. (2006b) find that $L_{\mathrm{H} 2 \mathrm{O}} \propto \sim L_{2-10}^{2}$, where $L_{2-10}$ is the $2-10 \mathrm{keV}$ X-ray luminosity of the host galaxy. Zhang et al. (2010, see also Henkel et al. 2005; Castangia et al. 2008; Surcis et al. 2009) find there appears to be a correlation between $L_{\mathrm{FIR}}$ and $L_{\mathrm{H} 2 \mathrm{O}}$, where $L_{\mathrm{FIR}}$ is the total far-infrared (FIR) luminosity of the host galaxy. However, they do not claim to find a significant correlation between extinction-corrected $L_{[\mathrm{OIII}] \lambda 5007 \text {, cor }}$ and $L_{\mathrm{H} 2 \mathrm{O}}$, likely due to the smaller sample size in their study.

\subsection{Masers favor high-extinction systems: a geometrical effect?}

In Figure 4, we showed that maser detection rate is higher at higher $\mathrm{H} \alpha / \mathrm{H} \beta$, thus higher extinction. Greenhill et al. (2008) and Zhang et al. (2010) also find that there is a high incidence of Compton-thick systems among AGN masers. If masers are only located on the nuclear disk surrounding the central black hole, then this preference of higher-extinction systems could be explained as a geometrical effect.

The specific intensity of maser emission scales with the cube of the pumping gain length $(l)$ along the path (e.g., Strelnitskii 1984; Lo 2005): $L_{\mathrm{H} 2 \mathrm{O}} \propto l^{3}$. If masers originate in gas clouds in the circumnuclear disk, we can only observe masers when the disk is inclined so that the gain length along the line-of-sight is long enough. According to the proposed unified AGN models (e.g., Antonucci \& Miller 1985), when the circumnuclear disk is inclined, the optical extinction due to the dusty torus and the X-ray obscuring column $\left(N_{H}\right)$ are correspondingly higher. Naturally, masers are more often found to be in high-extinction systems. Under this theory, we expect total isotropic maser luminosity on average to be higher in higher-extinction systems, since the average gain length should be longer. However, we did not find a convincing linear relation between $\log _{10} L_{\mathrm{H} 2 \mathrm{O}}$ and $\mathrm{H} \alpha / \mathrm{H} \beta$ in Figure 11. This could be because $\mathrm{H} \alpha / \mathrm{H} \beta$ is not a direct proxy of gain length, or the intrinsic scatter of the relation is too big to detect in the small sample we currently have. Meanwhile, some masers, e.g., NGC 1052 (Claussen et al. 1998) and NGC 262 (Mrk 348, Peck et al. 2003), are likely to be associated with jets but not disks. These systems could have greatly increased the scatter of the relation. It is also possible that the inclination of the host galaxy can affect the observed Balmer decrement, and thus increase the scatter.
On the other hand, if this theory is correct, then many extragalactic nuclear masers should be disk systems, but more than half of current maser detections lack evidence of association with disks (or jets). To confirm this theory requires further investigations with larger samples and follow-up VLBI observations of maser detections.

\section{SUGGESTIONS ON SURVEY STRATEGIES}

Our results suggest that if we can improve the observational sensitivity, we should be able to detect more nuclear masers. Given the current sensitivity, however, we can still improve the detection efficiency. Since there exist large optical spectroscopic surveys, such as SDSS, $2 \mathrm{dFGRS}$, and $6 \mathrm{dFGS}$, we suggest that maser surveys primarily interested in efficiency should select AGN targets from these surveys and rank them by extinctioncorrected [O III] $\lambda 5007$ flux. As an example, we illustrate this strategy in Figure 12

In Figure 12, we plot $\log _{10} L_{[\mathrm{OIII}] \lambda 5007}$ against redshift for all the 33 detections and 1030 non-detections with SDSS spectroscopy. Note in Figure 9, we showed that $0.1 \mathrm{Jy} \mathrm{km} \mathrm{s}^{-1}$ represents the effective flux limit in maser surveys. To relate this limit to $L_{[\mathrm{OIII}] \lambda 5007}$, we use the dotted lines in Figure 10. $\log _{10} L_{\mathrm{H} 2 \mathrm{O}}=$ $-39.6+\log _{10} L_{[\mathrm{OIII}] \lambda 5007, \operatorname{cor}}$ and $\log _{10} L_{\mathrm{H} 2 \mathrm{O}}=-38.6+$ $\log _{10} L_{[\mathrm{OIII}] \lambda 5007, \text { obs }}$, where $L_{\mathrm{H} 2 \mathrm{O}}$ is in $L_{\odot}$ and $L_{[\mathrm{OIII}] \lambda 5007}$ is in $\mathrm{erg} \mathrm{s}^{-1}$. This conversion translates the limit 0.1 Jy km s${ }^{-1}$ in maser flux into $7.6 \times 10^{-14} \mathrm{erg} \mathrm{s}^{-1} \mathrm{~cm}^{-2}$ in extinction-corrected [O III] $\lambda 5007$ flux, and $7.6 \times$ $10^{-15} \mathrm{erg} \mathrm{s}^{-1} \mathrm{~cm}^{-2}$ in observed [O III] $\lambda 5007$ flux. These limits are the solid lines in Figure 10. To take into account the scatter in the $L_{\mathrm{H} 2 \mathrm{O}}-L_{[\mathrm{OIII}] \lambda 5007}$ relation, we shift the limits downward by -1.0 dex to $7.6 \times 10^{-15} \mathrm{erg} \mathrm{s}^{-1} \mathrm{~cm}^{-2}$ and $7.6 \times 10^{-16} \mathrm{erg} \mathrm{s}^{-1} \mathrm{~cm}^{-2}$ and show them with the dashed lines. Among the 33 detections, 30 (32) have $L_{[\mathrm{OIII}] \lambda 5007 \text {,cor }}\left(L_{[\mathrm{OIII}] \lambda 5007, \mathrm{obs}}\right)$ brighter than the dashed line. For $L_{[\mathrm{OIII}] \lambda 5007 \text {, cor }}$ $\left(L_{[\mathrm{OIII}] \lambda 5007, \mathrm{obs}}\right)$, the detection rates above and below the dashed line are $6.9 \% \pm 1.2 \%(4.3 \% \pm 0.8 \%)$ and $0.5 \% \pm 0.3 \%(0.3 \% \pm 0.3 \%)$; the detection rates above and below the solid line are $16.0 \% \pm 4.1 \%(9.2 \% \pm 2.2 \%)$ and $2.0 \pm 0.5 \%(1.9 \% \pm 0.5 \%)$.

Therefore, if we rank AGN targets by extinctioncorrected [O III] $\lambda 5007$ flux, we can significantly improve the detection efficiency, from an overall $\sim 3 \%$ to $\sim 16 \%$ for the strongest [O III] $\lambda 5007$ emitters. Furthermore, given the current sensitivity, observing AGNs with extinction corrected [O III] $\lambda 5007$ flux lower than the dashed line yields a very low detection rate of $\lesssim 1 \%$. The contrast among these detection rates suggests that ranking source lists according to extinction-corrected [O III] $\lambda 5007$ is effective in maximizing detection efficiency. If extinction correction (i.e., $\mathrm{H} \alpha / \mathrm{H} \beta$ ) is not available, we suggest that maser surveys rank AGN targets with the observed [O III] $\lambda 5007$ flux. If the observed [O III] $\lambda 5007$ flux is not available either, we suggest that maser surveys rank AGN targets by velocity dispersion, or by optical luminosity. This strategy should give a higher detection efficiency than a blind survey.

We are grateful to Jim Braatz for allowing us to use the compilation of the galaxy sample surveyed for water maser emission before publication. We thank 


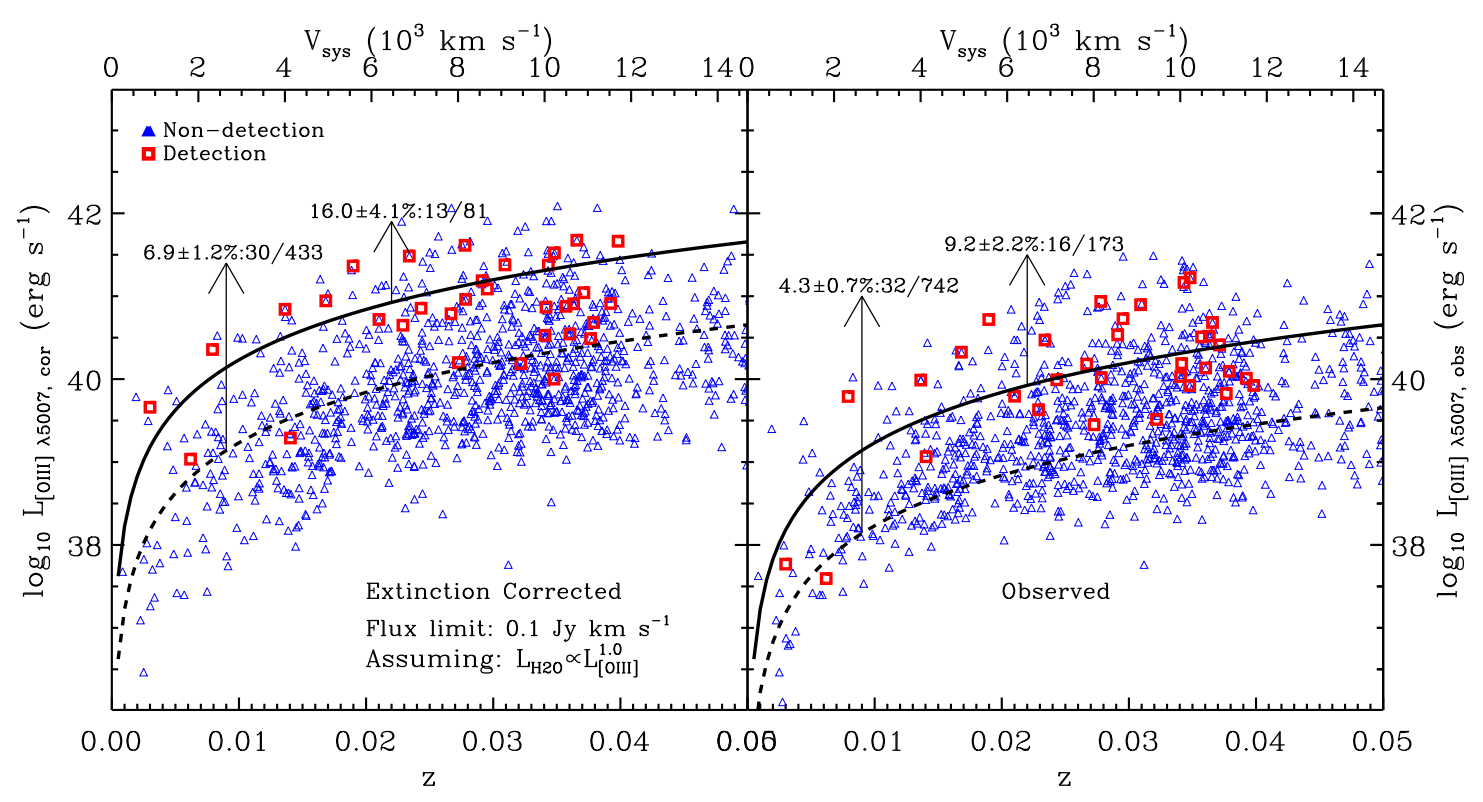

FIG. 12. - [O III $] \lambda 5007$ luminosity as a function of redshift. We plot all detections and non-detections in the SDSS spectroscopic sample. The solid lines show the assumption of maser flux limit $0.1 \mathrm{Jy} \mathrm{km} \mathrm{s}^{-1}$ and $L_{\mathrm{H} 2 \mathrm{O}} \propto L_{[\mathrm{OIII}] \lambda 5007}$ (the dotted lines in Figure 10). The dashed lines are the flux limit shifted downward by $-1.0 \mathrm{dex}$, to take into account the scatter in the $L_{\mathrm{H} 2 \mathrm{O}-} L_{[\mathrm{O} I I I]} \lambda 5007$ relation. The percentages at the end of arrows show the detection rates above the corresponding lines and the two numbers show the number of detections and galaxies surveyed for maser emission (detections plus non-detections).

David W. Hogg and an anonymous referee for comments that helped improve the manuscript. This research has made use of NASAs Astrophysics Data System and of the NASA/IPAC Extragalactic Database (NED) which is operated by the Jet Propulsion Laboratory, California Institute of Technology, under contract with the National Aeronautics and Space Administration. We also acknowledge the usage of the HyperLEDA database (http://leda.univ-lyon1.fr)

The authors acknowledge funding support from NSF grant AST-0607701, NASA grants 06-FLEX06-0030,
NNX09AC85G and NNX09AC95G, and Spitzer grant G05-AR-50443.

Funding for the SDSS and SDSS-II has been provided by the Alfred P. Sloan Foundation, the Participating Institutions, the National Science Foundation, the U.S. Department of Energy, the National Aeronautics and Space Administration, the Japanese Monbukagakusho, the Max Planck Society, and the Higher Education Funding Council for England. The SDSS Web Site is http://www.sdss.org/.

\section{REFERENCES}

Abazajian, K. N., et al. 2009, ApJS, 182, 543

Adelman-McCarthy, J. K., et al. 2006, ApJS, 162, 38

Antonucci, R. R. J., \& Miller, J. S. 1985, ApJ, 297, 621

Baldwin, J. A., Phillips, M. M., \& Terlevich, R. 1981, PASP, 93, 5

Balzano, V. A. 1983, ApJ, 268, 602

Bassani, L., Dadina, M., Maiolino, R., Salvati, M., Risaliti, G., della Ceca, R., Matt, G., \& Zamorani, G. 1999, ApJS, 121, 473

Blanton, M. R., \& Roweis, S. 2007, AJ, 133, 734

Blanton, M. R., et al. 2005, AJ, 129, 2562

Braatz, J. A., \& Gugliucci, N. E. 2008, ApJ, 678, 96

Braatz, J. A., Henkel, C., Greenhill, L. J., Moran, J. M., \& Wilson, A. S. 2004, ApJ, 617, L29

Braatz, J. A., Reid, M. J., Humphreys, E. M. L., Henkel, C., Condon, J. J., \& Lo, K. Y. 2010, ApJ, 718, 657

Braatz, J. A., Wilson, A. S., \& Henkel, C. 1996, ApJS, 106, 51 -. 1997, ApJS, 110, 321

Braatz, J. A., Wilson, A. S., Henkel, C., Gough, R., \& Sinclair, M. 2003, ApJS, 146, 249

Bragg, A. E., Greenhill, L. J., Moran, J. M., \& Henkel, C. 2000, ApJ, 535, 73

Castangia, P., Tarchi, A., Henkel, C., \& Menten, K. M. 2008, A\&A, 479, 111

Claussen, M. J., Diamond, P. J., Braatz, J. A., Wilson, A. S., \& Henkel, C. 1998, ApJ, 500, L129+

Colless, M., et al. 2001, MNRAS, 328, 1039
Corwin, Jr., H. G., Buta, R. J., \& de Vaucouleurs, G. 1994, AJ, 108,2128

Dahari, O., \& De Robertis, M. M. 1988, ApJS, 67, 249

de Vaucouleurs, G., de Vaucouleurs, A., Corwin, Jr., H. G., Buta, R. J., Paturel, G., \& Fouque, P. 1991, Third Reference Catalogue of Bright Galaxies

Diamond-Stanic, A. M., Rieke, G. H., \& Rigby, J. R. 2009, ApJ, 698,623

Elitzur, M., ed. 1992, Astrophysics and Space Science Library,

Vol. 170, Astronomical masers

Ferrarese, L., \& Merritt, D. 2000, ApJ, 539, L9

Frank, King, \& Raine, ed. 2002, Accretion Power in Astrophysics: Third Edition

Gebhardt, K., et al. 2000, ApJ, 539, L13

Gerssen, J., van der Marel, R. P., Axon, D., Mihos, J. C.,

Hernquist, L., \& Barnes, J. E. 2004, AJ, 127, 75

Greene, J. E., \& Ho, L. C. 2006, ApJ, 641, L21

Greenhill, L. J., Ellingsen, S. P., Norris, R. P., Gough, R. G., Sinclair, M. W., Moran, J. M., \& Mushotzky, R. 1997, ApJ, 474, L103+

Greenhill, L. J., \& Gwinn, C. R. 1997, Ap\&SS, 248, 261

Greenhill, L. J., Tilak, A., \& Madejski, G. 2008, ApJ, 686, L13

Greenhill, L. J., et al. 2003, ApJ, 590, 162

Gu, Q., Melnick, J., Cid Fernandes, R., Kunth, D., Terlevich, E., \& Terlevich, R. 2006, MNRAS, 366, 480

Gültekin, K., et al. 2009, ApJ, 698, 198 
Heckman, T. M., Kauffmann, G., Brinchmann, J., Charlot, S., Tremonti, C., \& White, S. D. M. 2004, ApJ, 613, 109

Heckman, T. M., Ptak, A., Hornschemeier, A., \& Kauffmann, G. 2005, ApJ, 634, 161

Henkel, C., Braatz, J. A., Tarchi, A., Peck, A. B., Nagar, N. M., Greenhill, L. J., Wang, M., \& Hagiwara, Y. 2005, Ap\&SS, 295, 107

Herrnstein, J. R., Moran, J. M., Greenhill, L. J., \& Trotter, A. S. 2005, ApJ, 629, 719

Ho, L. C., Filippenko, A. V., \& Sargent, W. L. W. 1997, ApJ, 487, 568

—. 2003, ApJ, 583, 159

Ho, L. C., Greene, J. E., Filippenko, A. V., \& Sargent, W. L. W. 2009, ApJS, 183, 1

Ishihara, Y., Nakai, N., Iyomoto, N., Makishima, K., Diamond, P., \& Hall, P. 2001, PASJ, 53, 215

Jones, D. H., et al. 2004, MNRAS, 355, 747

Kauffmann, G., et al. 2003, MNRAS, 346, 1055

Kewley, L. J., Dopita, M. A., Sutherland, R. S., Heisler, C. A., \& Trevena, J. 2001, ApJ, 556, 121

Kondratko, P. T., Greenhill, L. J., \& Moran, J. M. 2005, ApJ, 618,618

-. 2006a, ApJ, 652, 136

Kondratko, P. T., et al. 2006b, ApJ, 638, 100

Kuo, C. Y., et al. 2010, ArXiv e-prints

Lo, K. Y. 2005, ARA\&A, 43, 625

Madejski, G., Done, C., Życki, P. T., \& Greenhill, L. 2006, ApJ, 636,75

Magorrian, J., et al. 1998, AJ, 115, 2285

McElroy, D. B. 1995, ApJS, 100, 105
Miyoshi, M., Moran, J., Herrnstein, J., Greenhill, L., Nakai, N., Diamond, P., \& Inoue, M. 1995, Nature, 373, 127

Nelson, C. H., \& Whittle, M. 1995, ApJS, 99, 67

Neufeld, D. A., \& Maloney, P. R. 1995, ApJ, 447, L17+

Oliva, E., Origlia, L., Kotilainen, J. K., \& Moorwood, A. F. M. 1995, A\&A, 301, 55

Oliva, E., Origlia, L., Maiolino, R., \& Moorwood, A. F. M. 1999, A\&A, 350, 9

Peck, A. B., Henkel, C., Ulvestad, J. S., Brunthaler, A., Falcke, H., Elitzur, M., Menten, K. M., \& Gallimore, J. F. 2003, ApJ, 590, 149

Polletta, M., Bassani, L., Malaguti, G., Palumbo, G. G. C., \& Caroli, E. 1996, ApJS, 106, 399

Sarzi, M., et al. 2010, MNRAS, 402, 2187

Schlegel, D. J., Finkbeiner, D. P., \& Davis, M. 1998, ApJ, 500, 525

Strelnitskii, V. S. 1984, MNRAS, 207, 339

Surcis, G., Tarchi, A., Henkel, C., Ott, J., Lovell, J., \& Castangia, P. 2009, A\&A, 502, 529

Tremaine, S., et al. 2002, ApJ, 574, 740

Tremonti, C. A., et al. 2004, ApJ, 613, 898

Veilleux, S., \& Osterbrock, D. E. 1987, ApJS, 63, 295

Wegner, G., et al. 2003, AJ, 126, 2268

Whittle, M. 1992, ApJS, 79, 49

York, D. G., et al. 2000, AJ, 120, 1579

Zhang, J. S., Henkel, C., Guo, Q., Wang, H. G., \& Fan, J. H. 2010, ApJ, 708, 1528

Zhang, J. S., Henkel, C., Kadler, M., Greenhill, L. J., Nagar, N., Wilson, A. S., \& Braatz, J. A. 2006, A\&A, 450, 933 
Hosts of Water Masers

TABLE 1

Physical Parameters of EXtragalactic Water maser SOURCES

\begin{tabular}{|c|c|c|c|c|c|c|c|c|c|c|}
\hline Source & $\mathrm{V}_{\mathrm{sys}}^{1}$ & $\mathrm{~L}_{\mathrm{H} 2 \mathrm{O}^{2}}{ }^{2}$ & $\operatorname{Ref}\left(\mathrm{L}_{\mathrm{H} 2 \mathrm{O}}\right)^{3}$ & $\left.\mathrm{~F}_{[\mathrm{OIII}]}\right]^{4}$ & $\mathrm{H}_{\alpha} / \mathrm{H}_{\beta}{ }^{5}$ & $\operatorname{Ref}\left(\mathrm{F}_{[\mathrm{OIII}]}\right)^{6}$ & $\sigma^{7}$ & $\operatorname{Ref}(\sigma)^{8}$ & $\mathrm{M}_{B}^{9}$ & $\operatorname{Ref}\left(\mathrm{M}_{B}\right)^{10}$ \\
\hline NGC 23 (MrK 545) & 4566 & 2.3 & BG08 & 1200 & 5.2 & DD88 & $\ldots$ & $\ldots$ & -21.50 & DeV91 \\
\hline NGC $17\left(\right.$ Mrk 938) ${ }^{\dagger}$ & 5881 & $\ldots$ & $\ldots$ & $\ldots$ & $\ldots$ & $\ldots$ & $\ldots$ & $\ldots$ & & \\
\hline 2MASX J00114518-0054303 & 14384 & $\ldots$ & & $\ldots$ & $\ldots$ & $\ldots$ & $\ldots$ & $\ldots$ & -19.58 & SDSS \\
\hline NGC 235A & 6664 & 2.0 & Kondratko06 & $\ldots$ & t & $\ldots$ & 198 & Wegner03 & -21.02 & DeV91 \\
\hline NGC 262 (Mrk 348) & 4507 & 2.6 & Henkel05 & 22710 & 6.02 & Bassani99 & 185 & McElroy95 & -20.54 & DeV91 \\
\hline NGC 291 & 5705 & $\ldots$ & $\ldots$ & 6431 & 4.97 & SDSS & 109 & SDSS & -20.14 & SDSS \\
\hline ESO 013-G012 & 5047 & $\ldots$ & $\ldots$ & $\ldots$ & $\ldots$ & $\ldots$ & $\ldots$ & $\ldots$ & $\ldots$ & $\ldots$ \\
\hline NGC $449(\text { Mrk } 1)^{\dagger}$ & 4780 & 1.7 & Henkel05 & 60000 & & Whittle92 & 115 & NW95 & -19.53 & DeV91 \\
\hline NGC 520 & 2281 & 0.1 & Castangia08 & 412.4 & 4.13 & Ho97 & 41 & Ho09 & -20.55 & DeV91 \\
\hline 2MASX J01260163-0417564 & 5639 & $\ldots$ & & $\ldots$ & $\ldots$ & & $\ldots$ & & & \\
\hline NGC $591(\operatorname{MrK} 1157)^{\dagger}$ & 4547 & 1.4 & Henkel05 & 23000 & $\ldots$ & Whittle92 & 95 & NW95 & -20.50 & DeV91 \\
\hline NGC 613 & 1481 & $\begin{array}{l}1.2 \\
1.2\end{array}$ & $\begin{array}{l}\text { Kondratko06 } \\
\text { Castangia08 }\end{array}$ & $\ldots$ & $\begin{array}{l}\cdots \\
\cdots\end{array}$ & $\ldots$ & 125 & McElroy95 & -21.08 & DeV91 \\
\hline IC 0184 & 5382 & 1.4 & Kondratko06 & $\ldots$ & $\ldots$ & $\ldots$ & $\ldots$ & $\ldots$ & -20.00 & DeV91 \\
\hline 2MASX J02140591-0016371 & 11205 & $\ldots$ & $\ldots$ & $\cdots$ & $\cdots$ & $\cdots$ & $\cdots$ & $\cdots$ & -18.59 & SDSS \\
\hline Mrk 1029 & 9076 & & & $\ldots$ & & & & $\ldots$ & & \\
\hline NGC 1052 & 1510 & 2.1 & Henkel05 & 33068 & $2.35^{\mathrm{b}}$ & Ho97 & 187 & Wegner03 & -19.85 & SDSS \\
\hline NGC $1068(\text { M 77) })^{\dagger}$ & 1137 & $\begin{array}{l}2.2 \\
1.7\end{array}$ & $\begin{array}{l}\text { Henkel05 } \\
\text { KGM06 }\end{array}$ & 3497963 & 5.29 & Ho97 & 162 & Ho09 & -21.68 & DeV91 \\
\hline NGC 1106 & 4337 & 0.9 & BG08 & & & & 146 & Wegner03 & -21.17 & DeV91 \\
\hline 2MASX J02532956-0014052 & 8622 & $\ldots$ & & 1757 & 5.0 & SDSS & 96 & SDSS & -18.18 & SDSS \\
\hline Mrk 1066 & 3605 & 1.5 & Henkel05 & 24000 & 8.51 & Whittle92 & 105 & NW95 & -20.60 & DeV91 \\
\hline NGC $1194^{\dagger}$ & 4076 & $\ldots$ & $\ldots$ & 2340 & 5.85 & SDSS & 144 & SDSS & -20.06 & SDSS \\
\hline NGC $1320(\mathrm{MrK} 607)^{\dagger}$ & 2663 & $\ldots$ & $\ldots$ & $\ldots$ & $\ldots$ & $\ldots$ & $\ldots$ & $\ldots$ & $\ldots$ & $\ldots$ \\
\hline 2MASX J03364614-0750236 & 11719 & $\ldots$ & $\ldots$ & $\ldots$ & $\ldots$ & $\ldots$ & $\ldots$ & $\ldots$ & $\ldots$ & $\ldots$ \\
\hline NGC $1386^{\dagger}$ & 868 & 2.1 & Henkel05 & 99244 & 5.7 & Bassani99 & 187 & McElroy95 & -18.52 & DeV91 \\
\hline $\begin{array}{l}\text { 2MASX J03381036+0114178 } \\
\text { (IRAS03355+0104) }\end{array}$ & 11926 & $\ldots$ & $\ldots$ & $\ldots$ & $\ldots$ & $\ldots$ & $\ldots$ & $\ldots$ & $\ldots$ & $\ldots$ \\
\hline $\begin{array}{l}4 \mathrm{C}+05.19 \\
(2 \mathrm{MASX} \text { J04143774+0534423) }\end{array}$ & 790000 & $\cdots$ & $\cdots$ & $\cdots$ & $\cdots$ & $\ldots$ & $\cdots$ & $\cdots$ & $\cdots$ & $\cdots$ \\
\hline $\begin{array}{l}\text { 2MASX J04405494-0822221 } \\
\text { (IRAS F04385-0828) }\end{array}$ & 4527 & $\cdots$ & $\cdots$ & $\cdots$ & $\cdots$ & $\cdots$ & $\cdots$ & $\cdots$ & $\cdots$ & $\cdots$ \\
\hline $\mathrm{UGC} 3193^{\dagger}$ & 4454 & 2.4 & BG08 & $\ldots$ & $\ldots$ & $\ldots$ & $\ldots$ & $\ldots$ & -19.93 & DeV91 \\
\hline NGC 1741 (Mrk 1089) & 4039 & $\ldots$ & $\ldots$ & $\ldots$ & $\ldots$ & $\ldots$ & $\ldots$ & $\ldots$ & $\ldots$ & $\ldots$ \\
\hline CGCG 468-002 & 5454 & & & $\ldots$ & $\ldots$ & $\ldots$ & $\ldots$ & $\ldots$ & & \\
\hline UGC 3255 & 5669 & 1.2 & Henkel05 & $\ldots$ & $\ldots$ & $\ldots$ & $\ldots$ & $\ldots$ & -19.98 & DeV91 \\
\hline UGCA 116 & 789 & $\ldots$ & & $\ldots$ & & $\ldots$ & $\ldots$ & $\ldots$ & & \\
\hline Mrk 3 & 4050 & 1.0 & Henkel05 & 439100 & 6.67 & Bassani99 & 248 & McElroy95 & -20.70 & DeV91 \\
\hline VII Zw 073 & 12391 & 2.2 & Kondratko06 & $\ldots$ & & & $\ldots$ & $\ldots$ & $\ldots$ & \\
\hline NGC 2273 (MrK 620) & 1840 & 0.8 & Zhang06 & 27618 & 5.08 & Ho97 & 149 & Ho09 & -19.95 & DeV91 \\
\hline UGC $3789^{\dagger}$ & 3325 & 2.6 & BG08 & $\ldots$ & & & $\ldots$ & $\ldots$ & -20.47 & DeV91 \\
\hline NGC 2410 & 4681 & & & 3306 & 4.88 & SDSS & 166 & SDSS & -20.86 & SDSS \\
\hline Mrk 78 & 11137 & 1.5 & Henkel05 & 66000 & 6.46 & Polletta96 & 114 & McElroy95 & & \\
\hline IC $0485^{\dagger}$ & 8338 & & & 585.8 & 6.29 & SDSS & 187 & SDSS & -20.24 & SDSS \\
\hline Mrk 1210 (UGC 04203, Phoenix) & 4046 & 1.9 & Henkel05 & 95660 & 5.2 & Bassani99 & 114 & $\mathrm{Gu} 06$ & -19.71 & DeV91 \\
\hline SDSS J0804+3607 & 198000 & $\ldots$ & $\ldots$ & $\ldots$ & $\ldots$ & $\ldots$ & $\ldots$ & $\ldots$ & $\ldots$ & $\ldots$ \\
\hline 2 MASX J08362280+3327383 ${ }^{\dagger}$ & 14810 & $\ldots$ & & $\ldots$ & $\ldots$ & $\ldots$ & $\ldots$ & $\ldots$ & -20.24 & SDSS \\
\hline NGC $2639^{\dagger}$ & 3336 & 1.4 & Henkel05 & 1858 & 4.06 & Ho97 & 179 & Ho09 & -20.95 & SDSS \\
\hline NGC 2781 & 2053 & $\ldots$ & $\ldots$ & 1078 & & & $\ldots$ & & & \\
\hline 2MASX J09124641+2304273 & 10861 & $\ldots$ & & 1078 & 4.07 & SDSS & 74 & SDSS & -19.12 & SDSS \\
\hline NGC 2782 & 2543 & 1.1 & Henkel05 & 5944 & 6.5 & Ho97 & 183 & Ho09 & -20.41 & SDSS \\
\hline NGC 2824 (MrK 394) & 2760 & 2.7 & Henkel05 & $\ldots$ & & & 122 & McElroy95 & -19.09 & SDSS \\
\hline SBS $0927+493$ & 10167 & & & 570.9 & 5.11 & SDSS & 147 & SDSS & -20.70 & SDSS \\
\hline UGC 5101 & 11802 & 3.2 & Zhang06 & 226.9 & 11.7 & SDSS & 189 & SDSS & -21.28 & SDSS \\
\hline NGC $2960\left(\right.$ MrK 1419) ${ }^{\dagger}$ & 4932 & 2.6 & Henkel05 & 4400 & $\ldots$ & DD88 & $\ldots$ & $\ldots$ & -20.84 & SDSS \\
\hline NGC $2979^{\dagger}$ & 2720 & 2.1 & Henkel05 & $\ldots$ & $\ldots$ & $\ldots$ & 112 & Gu06 & & \\
\hline NGC 2989 & 4146 & 1.6 & BG08 & $\ldots$ & $\ldots$ & $\ldots$ & $\ldots$ & $\ldots$ & -20.66 & DeV91 \\
\hline NGC 3081 & 2391 & $\ldots$ & $\ldots$ & $\ldots$ & $\ldots$ & $\ldots$ & $\ldots$ & $\ldots$ & $\ldots$ & $\ldots$ \\
\hline NGC $3079^{\dagger}$ & 1116 & $\begin{array}{l}2.7 \\
2.5\end{array}$ & $\begin{array}{c}\text { Henkel05 } \\
\text { Kondratko05 }\end{array}$ & 176.2 & 28.4 & Ho97 & 182 & Ho09 & -19.62 & DeV91 \\
\hline 2MASX J10115058-1926436 & 8065 & $\ldots$ & $\ldots$ & $\ldots$ & & & $\ldots$ & & & \\
\hline NGC 3160 & 6920 & $\ldots$ & & 358.5 & 6.66 & SDSS & 155 & SDSS & -20.32 & SDSS \\
\hline IC $2560^{\dagger}$ & 2925 & 2.0 & Henkel05 & $\ldots$ & $\ldots$ & $\ldots$ & 144 & $\mathrm{Gu} 06$ & -21.09 & DeV91 \\
\hline NGC 3256 & 2804 & 0.8 & Surcis09 & $\ldots$ & $\ldots$ & $\ldots$ & 127 & Oliva95 & -21.49 & DeV91 \\
\hline UGC $5713^{\dagger}$ & 6312 & $\ldots$ & 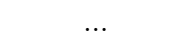 & 622.0 & 6.2 & SDSS & 168 & SDSS & -20.24 & SDSS \\
\hline Mrk $34^{\dagger}$ & 15140 & 3.0 & Henkel05 & 67000 & 10.5 & Polletta96 & $\ldots$ & $\ldots$ & $\ldots$ & $\ldots$ \\
\hline NGC $3393^{\dagger}$ & 3750 & $\begin{array}{l}2.4 \\
2.6\end{array}$ & $\begin{array}{l}\text { Kondratko06 } \\
\text { Zhang06 }\end{array}$ & 124344 & 4.12 & Bassani99 & 184 & McElroy95 & -20.99 & DeV91 \\
\hline UGC $6093^{\dagger}$ & 10828 & $\ldots$ & 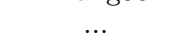 & 452.0 & 4.14 & SDSS & 160 & SDSS & -21.53 & SDSS \\
\hline 2MASX J11093314+2837393 & 11422 & $\ldots$ & $\ldots$ & 371.7 & 4.76 & SDSS & 149 & SDSS & -20.41 & SDSS \\
\hline NGC 3620 & 1680 & 0.5 & Surcis09 & $\ldots$ & & & $\ldots$ & & $\ldots$ & \\
\hline CGCG 185-028 & 10455 & $\ldots$ & 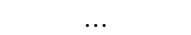 & 298.7 & 3.19 & SDSS & 204 & SDSS & -21.14 & SDSS \\
\hline
\end{tabular}


TABLE 1

NGC 3614

Arp $299($ NGC 3690 \& IC 694)a NGC $3735^{\dagger}$

CGCG 068-013

NGC 3783

CGCG 268-089

(MCG +09-19-205)

2MASX J12020465+3519173 ${ }^{\dagger}$

UGC $7016^{\dagger}$

NGC $4051^{\dagger}$

NGC 4151

NGC 4253 (MrK 766)

NGC $4258(\text { M 106) })^{\dagger}$

NGC 4293

NGC $4388^{\dagger}$

NGC 4527

NGC 4633 (IC 3688)

ESO 269-G012 ${ }^{\dagger}$

NGC $4922 \mathrm{~N}$

NGC $4945^{\dagger}$

NGC 4968

NGC 5194 (M 51A)

NGC 5256S (MrK 0266)

SBS $1344+527^{\dagger}$

NGC 5347

2MASX J13553592+0553050

(IRAS $13530+0607$ )

MCG +11-17-010

ESO 446-G018

NGC $5495^{\dagger}$

Circinus $^{\dagger}$

NGC 5506 (MrK 1376)

NGC 5643

NGC 5691

NGC $5728^{\dagger}$

CGCG 164-019

UGC $9618^{\dagger}$

MrK 834 (UGC 9639) $^{\dagger}$

NGC $5793^{\dagger}$

2MASX J15201964+5253560

2MASX J16070391+0106296

IRAS $16288+3929$

CGCG 168-018

NGC 6240 (IC 4625)

NGC $6264^{\dagger}$

2MFGC 13581

${ }^{2 M A S X}$ J16581548+3923294) ${ }^{\dagger}$

2MASX J17101815+1344058

(IRAS F17080+1347)

NGC $6323^{\dagger}$

NGC 6300

ESO 103-G035

2MASX J19393889-0124328

(IRAS F19370-0131)

$3 \mathrm{C} 403^{\dagger}$

NGC $6926^{\dagger}$

UGC 11685

IC 1361

AM 2158-380 NED02

2MASX J22291248-1810470

(IRAS F22265-1826)

NGC 7479

IC 1481

CGCG 498-038

Physical PARAMETERS OF EXTRAGALACTIC WATER MASER SOURCES

\begin{tabular}{|c|c|c|c|c|c|c|c|c|c|}
\hline 2333 & & & & & & & & -19.68 & SDSS \\
\hline $\begin{array}{l}3088 \\
2696\end{array}$ & $\begin{array}{l}2.4 \\
1.3\end{array}$ & Henkel05 & 4917 & 6.04 & $\begin{array}{l}\mathrm{Ho9} 97 \\
\mathrm{Ho} 97\end{array}$ & 144 & $\begin{array}{l}\text { Ho09 } \\
\text { Ho09 }\end{array}$ & $\begin{array}{c}\ldots \\
-20.60\end{array}$ & $\begin{array}{c}\cdots \\
\text { DeV91 }\end{array}$ \\
\hline $\begin{array}{c}2690 \\
10660\end{array}$ & $\begin{array}{l}1.3 \\
\ldots\end{array}$ & $\begin{array}{c}\text { Henkel05 } \\
\ldots\end{array}$ & $\begin{array}{l}3741 \\
1092\end{array}$ & $\begin{array}{c}6.31 \\
4.0\end{array}$ & $\begin{array}{l}\text { Ho97 } \\
\text { SDSS }\end{array}$ & $\begin{array}{l}141 \\
111\end{array}$ & $\begin{array}{l}\text { Ho09 } \\
\text { SDSS }\end{array}$ & $\begin{array}{l}-20.60 \\
-21.28\end{array}$ & $\begin{array}{l}\text { Dev91 } \\
\text { SDSS }\end{array}$ \\
\hline 2917 & $\ldots$ & $\ldots$ & & & & & & & \\
\hline 7924 & $\ldots$ & $\ldots$ & 930.5 & 4.83 & SDSS & 128 & SDSS & -20.22 & SDSS \\
\hline 10201 & $\ldots$ & $\ldots$ & 401.0 & 4.43 & SDSS & 105 & SDSS & -19.94 & SDSS \\
\hline 7271 & $\ldots$ & $\ldots$ & 728.6 & 4.89 & SDSS & 177 & SDSS & -20.83 & SDSS \\
\hline 700 & 0.3 & Henkel05 & 44009 & 3.3 & Ho97 & 89 & GH06 & -19.32 & DeV91 \\
\hline 995 & -0.2 & Henkel05 & 1125847 & 3.4 & Ho97 & 97 & GH06 & -18.75 & SDSS \\
\hline 3876 & $\ldots$ & $\ldots$ & 262.0 & 3.58 & SDSS & 85 & SDSS & -20.34 & SDSS \\
\hline 448 & 1.9 & Henkel05 & 10430 & 3.94 & Ho97 & 167 & McElroy95 & -20.09 & DeV91 \\
\hline 893 & 0.4 & Kondratko06 & 295.3 & 13.2 & SDSS & 92 & SDSS & -18.10 & SDSS \\
\hline 2524 & 1.1 & Henkel05 & 66226 & 5.69 & Ho97 & 92 & Ho09 & -21.14 & SDSS \\
\hline 1736 & 0.6 & BG08 & 46.30 & 9.27 & SDSS & 142 & SDSS & -19.82 & SDSS \\
\hline 291 & $\ldots$ & $\ldots$ & $\ldots$ & $\ldots$ & $\ldots$ & $\ldots$ & $\ldots$ & $\ldots$ & $\ldots$ \\
\hline 5014 & 3.0 & Henkel05 & & & & & & -20.31 & DeV91 \\
\hline 7071 & 2.3 & Henkel05 & 2379 & 6.63 & SDSS & 174 & SDSS & -21.42 & SDSS \\
\hline $\begin{array}{c}563 \\
2957\end{array}$ & 1.7 & Henkel05 & $\ldots$ & $\cdots$ & $\ldots$ & 134 & Oliva95 & -21.07 & DeV91 \\
\hline $\begin{array}{c}2957 \\
463\end{array}$ & -0.2 & Henkel05 & 11358 & 8.44 & Ho97 & 113 & McElroy95 & -20.38 & DeV91 \\
\hline 8353 & 1.5 & Henkel05 & 4902 & 5.09 & SDSS & 187 & SDSS & -21.06 & SDSS \\
\hline 8763 & & & 2675 & 3.97 & SDSS & 147 & SDSS & -20.04 & SDSS \\
\hline 2335 & 1.5 & Henkel05 & 4433 & 4.68 & SDSS & 90 & SDSS & -19.26 & SDSS \\
\hline 11776 & $\ldots$ & $\ldots$ & 285.9 & 6.08 & SDSS & 127 & SDSS & -20.03 & SDSS \\
\hline 9456 & $\ldots$ & $\ldots$ & 136.9 & 5.08 & SDSS & 129 & SDSS & -20.06 & SDSS \\
\hline 4771 & $\ldots$ & $\ldots$ & $\ldots$ & $\ldots$ & $\ldots$ & $\ldots$ & $\ldots$ & $\ldots$ & $\cdots$ \\
\hline 6737 & 2.3 & Kondratko06 & $\ldots$ & $\ldots$ & $\ldots$ & $\ldots$ & $\ldots$ & -21.78 & DeV91 \\
\hline 434 & 1.3 & Henkel05 & 30180 & 19.1 & Bassani99 & 168 & Oliva95 & & \\
\hline 1853 & 1.7 & Henkel05 & 45744 & 7.2 & Bassani99 & 180 & Oliva99 & -19.50 & SDSS \\
\hline 1199 & $\begin{array}{l}1.4 \\
1.3\end{array}$ & $\begin{array}{c}\text { Henkel05 } \\
\text { KGM06 }\end{array}$ & 74804 & 6.4 & Bassani99 & $\ldots$ & $\ldots$ & -21.20 & DeV91 \\
\hline 1870 & $\ldots$ & $\ldots$ & $\ldots$ & $\ldots$ & $\ldots$ & $\ldots$ & $\ldots$ & -19.69 & SDSS \\
\hline 2804 & 1.9 & Henkel05 & 68000 & $\ldots$ & Whittle92 & 209 & McElroy95 & -21.18 & DeV91 \\
\hline 8963 & $\ldots$ & $\ldots$ & $\ldots$ & $\ldots$ & $\ldots$ & $\ldots$ & $\ldots$ & -20.37 & SDSS \\
\hline 10103 & $\ldots$ & $\ldots$ & $\ldots$ & $\ldots$ & $\ldots$ & $\ldots$ & $\ldots$ & -21.16 & SDSS \\
\hline 10802 & $\ldots$ & $\ldots$ & 1554 & 6.52 & SDSS & 177 & SDSS & -21.79 & SDSS \\
\hline 3491 & 2.0 & Henkel05 & & & & & & -19.75 & DeV91 \\
\hline 11166 & $\ldots$ & $\ldots$ & 203.3 & 5.04 & SDSS & 107 & SDSS & -20.23 & SDSS \\
\hline 8216 & $\ldots$ & $\ldots$ & 165.5 & 5.4 & SDSS & 126 & SDSS & -19.47 & SDSS \\
\hline 9161 & $\ldots$ & $\ldots$ & 3595 & 4.37 & SDSS & 142 & SDSS & -20.33 & SDSS \\
\hline 11015 & $\ldots$ & $\ldots$ & 805.8 & 4.92 & SDSS & 118 & SDSS & -20.00 & SDSS \\
\hline 7339 & 1.6 & Henkel05 & 795 & 17.2 & Bassani99 & 200 & Gerssen04 & -21.76 & DeV91 \\
\hline 10177 & $\ldots$ & $\ldots$ & 5383 & 3.53 & SDSS & 149 & SDSS & -20.91 & SDSS \\
\hline 10290 & $\ldots$ & $\ldots$ & 5947 & 3.79 & SDSS & 122 & SDSS & -20.44 & SDSS \\
\hline 9448 & $\ldots$ & $\cdots$ & $\ldots$ & $\ldots$ & $\cdots$ & $\cdots$ & $\cdots$ & $\cdots$ & $\ldots$ \\
\hline 7772 & 2.7 & Henkel05 & & & & $\ldots$ & $\cdots$ & -20.58 & DeV91 \\
\hline 1109 & $\begin{array}{c}0.5 \\
0.34\end{array}$ & $\begin{array}{c}\text { Henkel05 } \\
\text { KGM06 }\end{array}$ & 1349 & 7.44 & Polletta96 & $\ldots$ & $\ldots$ & -20.53 & DeV91 \\
\hline 3983 & 2.6 & Henkel05 & 12589 & 6.31 & Polletta96 & 114 & Gu06 & -19.51 & DeV91 \\
\hline 6622 & 2.2 & Henkel05 & $\ldots$ & $\ldots$ & $\ldots$ & $\ldots$ & $\ldots$ & $\ldots$ & $\ldots$ \\
\hline 17688 & 3.3 & Henkel05 & $\ldots$ & $\ldots$ & $\ldots$ & $\ldots$ & $\ldots$ & $\ldots$ & $\ldots$ \\
\hline 5880 & 2.7 & Henkel05 & $\ldots$ & $\ldots$ & $\ldots$ & $\ldots$ & $\ldots$ & -22.29 & DeV91 \\
\hline 5872 & $\ldots$ & $\ldots$ & $\ldots$ & $\ldots$ & $\ldots$ & $\ldots$ & $\ldots$ & $\ldots$ & $\ldots$ \\
\hline 3962 & 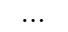 & & $\ldots$ & $\ldots$ & $\ldots$ & $\ldots$ & $\ldots$ & & \\
\hline 9983 & 2.7 & Kondratko06 & $\ldots$ & $\ldots$ & $\ldots$ & $\cdots$ & $\ldots$ & -21.24 & DeV91 \\
\hline 7520 & 3.8 & Henkel05 & $\ldots$ & $\ldots$ & $\ldots$ & $\ldots$ & $\ldots$ & $\ldots$ & $\ldots$ \\
\hline 2381 & 1.3 & BG08 & 1103 & 9.29 & Ho97 & 155 & Ho09 & -21.64 & DeV91 \\
\hline 6118 & 2.5 & Henkel05 & $\ldots$ & $\ldots$ & $\ldots$ & $\ldots$ & $\ldots$ & -20.77 & DeV91 \\
\hline 9240 & $\ldots$ & $\ldots$ & $\ldots$ & $\ldots$ & $\ldots$ & $\ldots$ & $\ldots$ & $\ldots$ & $\ldots$ \\
\hline
\end{tabular}


TABLE 1

Physical Parameters of EXTRAGalaCtic WATER MASER SOURCES

1 Heliocentric systemic velocity, in $\mathrm{km} \mathrm{s}^{-1}$.

2 Total maser luminosity assuming isotropic emission of radiation, defined as $L_{\mathrm{H} 2 \mathrm{O}}=0.023 \times \int S(V) d V \times D^{2}$, where $L_{\mathrm{H} 2 \mathrm{O}}$ is in $L_{\odot}, S(V)$ is the flux (in Jy) at velocity $V$ (in $\mathrm{km} \mathrm{s}^{-1}$ ), and $D$ is luminosity distance (in $\mathrm{Mpc}$ ). These values were calculated based on $H_{0}=75 \mathrm{~km} \mathrm{~s}^{-1}$ and We convert values into those with $H_{0}=70 \mathrm{~km} \mathrm{~s}^{-1}$ in the analysis.

3 References - Henkel05: Henkel et al. (2005); Kondratko05: Kondratko et al. (2005); Kondratko06: Kondratko et al. (2006a); KGM06: Kondratko et al. 2006b); Zhang06: Zhang et al. (2006); BG08: Braatz \& Gugliucci (2008); Castangia08: Castangia et al. (2008); Surcis09: Surcis et al. (2009).

4 Observed flux of [O III] $\lambda 5007$, in $10^{-17} \mathrm{erg} \mathrm{s}^{-1} \mathrm{~cm}^{-2}$.

5 Ratio of observed flux of $\mathrm{H} \alpha$ to that of $\mathrm{H} \beta$.

6 References - DD88: Dahari \& De Robertis (1988); Whittle92: Whittle (1992); Polletta96: Polletta et al. (1996); Ho97: Ho et al. (1997); Bassani99: Bassani et al. (1999). In the compilation, we first prefer the homogeneous samples from SDSS and Ho97. We then choose the most recent values for the rest of the sample.

7 Velocity Dispersion $\sigma$, in $\mathrm{km} \mathrm{s}^{-1}$.

8 References - McElroy95: McElroy (1995); NW95: Nelson \& Whittle (1995); Oliva95: Oliva et al. (1995); Oliva99: Oliva et al. (1999); Wegner03: Wegner et al. (2003); Gerssen04: Gerssen et al. (2004); Gu06: Gu et al. (2006); GH06: Greene \& Ho (2006); Ho09: Ho et al. (2009).

9 band absolute magnitude.

10 References - DeV91: de Vaucouleurs et al. (1991). Magnitudes are corrected for foreground Galatic extinction (Schlegel et al. 1998).

a The merging system Arp 299 consists of two galaxies, NGC 3690 and IC 694 . We assume the maser is associated with NGC 3690.

b Assumed to be 3 in the analysis.

$\dagger$ Likely disk systems. 